\title{
ON THE IMPLEMENTATION OF MIXED METHODS AS NONCONFORMING METHODS FOR SECOND-ORDER ELLIPTIC PROBLEMS
}

\author{
TODD ARBOGAST AND ZHANGXIN CHEN
}

\begin{abstract}
In this paper we show that mixed finite element methods for a fairly general second-order elliptic problem with variable coefficients can be given a nonmixed formulation. (Lower-order terms are treated, so our results apply also to parabolic equations.) We define an approximation method by incorporating some projection operators within a standard Galerkin method, which we call a projection finite element method. It is shown that for a given mixed method, if the projection method's finite element space $M_{h}$ satisfies three conditions, then the two approximation methods are equivalent. These three conditions can be simplified for a single element in the case of mixed spaces possessing the usual vector projection operator. We then construct appropriate nonconforming spaces $M_{h}$ for the known triangular and rectangular elements. The lowest-order Raviart-Thomas mixed solution on rectangular finite elements in $\mathbb{R}^{2}$ and $\mathbb{R}^{3}$, on simplices, or on prisms, is then implemented as a nonconforming method modified in a simple and computationally trivial manner. This new nonconforming solution is actually equivalent to a postprocessed version of the mixed solution. A rearrangement of the computation of the mixed method solution through this equivalence allows us to design simple and optimal-order multigrid methods for the solution of the linear system.
\end{abstract}

\section{INTRODUCTION}

We consider the following elliptic problem for $u$ on the bounded domain $\Omega \subset \mathbb{R}^{n}, n=2$ or 3 , with boundary $\partial \Omega=\bar{\Gamma}_{1} \cup \bar{\Gamma}_{2}, \Gamma_{1} \cap \Gamma_{2}=\varnothing$ :

$$
\begin{aligned}
& \nabla \cdot \sigma+d u=f \quad \text { in } \Omega, \\
& \sigma=-a(\nabla u+b u-c) \text { in } \Omega, \\
& u=-g \text { on } \Gamma_{1}, \\
& \sigma \cdot \nu=0 \text { on } \Gamma_{2},
\end{aligned}
$$

where $a(x)$ is a uniformly positive definite, bounded, symmetric tensor, $b(x)$ and $c(x)$ are bounded vectors, $d(x) \geq 0$ is bounded, $f(x) \in L^{2}(\Omega), g(x) \in$

Received by the editor August 23, 1993 and, in revised form, May 7, 1994.

1991 Mathematics Subject Classification. Primary 65N30, 65N22.

Key words and phrases. Finite element, implementation, mixed method, equivalence, nonconforming method, multigrid method.

The first author was supported in part by the National Science Foundation and the State of Texas Governor's Energy Office. The second author was supported in part by the Army Research Office contract number DAAL03-89-C-0038 with the University of Minnesota Army High Performance Computing Research Center. 
$H^{2}(\Omega)\left(H^{k}(\Omega)=W^{k, 2}(\Omega)\right.$ is the Sobolev space of $k$ times differentiable functions in $\left.L^{2}(\Omega)\right)$, and $\nu$ is the outer unit normal to the domain. Let $(\cdot, \cdot)_{S}$ denote the $L^{2}(S)$ inner product (we omit $S$ if $S=\Omega$ ). Assume that the problem is coercive in the sense that there is a positive constant $\kappa$ such that for any $v \in\left(L^{2}(\Omega)\right)^{n}$ and $w \in L^{2}(\Omega)$,

$$
\left(a^{-1} v, v\right)+(b w, v)+(d w, w) \geq \kappa\left\{\|v\|_{\left(L^{2}(\Omega)\right)^{n}}^{2}+(d w, w)\right\}
$$

(this immediately implies that if $d=0$ a.e. on a set $S$, then $b=0$ a.e. on $S$ ). Assume also that if $\Gamma_{1}=\varnothing$, then $d>0$ on some set of positive measure, so that if $v=-a(\nabla w+b w-c)$, then a generalized Poincaré inequality gives us control over $w$.

Problem (1.1) is recast in mixed form as follows. Let

$$
\begin{aligned}
& H(\operatorname{div} ; \Omega)=\left\{v \in\left(L^{2}(\Omega)\right)^{n}: \nabla \cdot v \in L^{2}(\Omega)\right\}, \\
& V=\left\{v \in H(\operatorname{div} ; \Omega): v \cdot \nu=0 \text { on } \Gamma_{2}\right\}, \\
& W=L^{2}(\Omega) .
\end{aligned}
$$

Then the mixed form of (1.1) for the pair $(\sigma, u) \in V \times W$ is

$$
\begin{aligned}
& (\nabla \cdot \sigma, w)+(d u, w)=(f, w), \quad \forall w \in W, \\
& \left(a^{-1} \sigma, v\right)-(u, \nabla \cdot v)+(b u, v)=(c, v)+(g, v \cdot \nu)_{\Gamma_{1}}, \quad \forall v \in V .
\end{aligned}
$$

In 1985, Arnold and Brezzi [1] showed that if $b=c=d=0$, and $n=2$, the mixed finite element methods for the even-order Raviart-Thomas spaces defined over triangles are equivalent to certain nonconforming methods. In particular, the lowest-order Raviart-Thomas space defined over triangles [21] is equivalent to a simple modification of the $P_{1}$-nonconforming Galerkin method. This nonconforming method yields a symmetric and positive definite problem (i.e., a minimization problem), whereas the mixed formulation is a saddle point problem.

Marini [18] noted that the computational cost of this modification is almost nil, if $a$ is a piecewise constant scalar. This equivalence has been exploited to obtain optimal $L^{\infty}(\Omega)$-error estimates for the mixed method [16]. Recently, Brenner [4] has used the equivalence to define and analyze an optimally convergent multigrid method. Chen $[9,11]$ has derived some nonconforming methods that are equivalent to certain lower-dimensional mixed methods, and exploited superconvergence properties to obtain a better approximation to the scalar variable.

Analogous equivalences for problems with nonzero low-order terms or for problems posed in higher dimensions (say $n=3$ ) have not been shown. It is necessary to obtain an equivalence for $d \not \equiv 0$ to treat time-dependent, parabolic problems. Moreover, an equivalence has not been shown for rectangular mixed methods, even though they are used widely in practice. We consider such problems in this paper, concentrating on the case of the lowest-order Raviart-Thomas mixed method defined over rectangles or rectangular parallelepipeds. An outline of the paper and a summary of our results follows.

We begin in $\S 2$ with the development of a general theory on the equivalence of mixed and nonconforming methods. Our theory is similar to, but more general than, that developed earlier by one of the authors [11]. We generalize the results of Arnold and Brezzi [1] in defining a nonconforming method for some 
finite element space $M_{h}$. It is a Galerkin method with the addition of some special projection operators, and hence we will call it a projection finite element method. We then develop three conditions on $M_{h}$ that are sufficient to imply the equivalence of the projection method to a given mixed method. In $\S 3$ we consider the problem of constructing finite element spaces that satisfy these three conditions. We derive a simple local criterion that guarantees the equivalence in the case of mixed spaces possessing the usual vector projection operator. In $\S \S 4$ and 5 , we use this general theory to define equivalent projection methods for various mixed methods for the problem (1.3). We treat the mixed spaces of Raviart and Thomas [21], Nedelec [19], Brezzi, Douglas, and Marini [8], Brezzi, Douglas, Durán, and Fortin [6], and Brezzi, Douglas, Fortin, and Marini [7] defined over triangles or rectangular parallelepipeds in $\mathbb{R}^{2}$ and $\mathbb{R}^{3}$. Our nonconforming spaces perhaps illuminate some of the relationships between these mixed spaces. We point out that projection finite element spaces are not necessarily unique, since two such spaces are known for the lowest-order Raviart-Thomas space over triangles: the one defined by Arnold and Brezzi [1] uses cubic "bubble functions" while the one defined by Chen [11] uses quadratic bubble functions.

Then, for several sections, we restrict our attention to the lowest-order Raviart-Thomas mixed method on rectangles. In $\S 6$, our general projection space is shown to have a nice structure. It is a simple augmentation of a standard nonconforming Galerkin space with $P_{2}$-bubble functions. These bubble functions are orthogonal in some sense to the standard nonconforming part of the solution. Diagonal $a$ and a modification to the mixed method, in which the coefficients are projected into the space of piecewise constants, allows us to exploit this fact. We can therefore give an explicit expression for the bubble function corrections (see formula (6.9) below), and so the method is easily implemented. A trivial postprocessing of its solution recovers the mixed solution. However, the nonconforming solution has better convergence properties than the mixed solution in that the scalar variable is approximated to the optimal order two (see §7). Alternatively, we may view the nonconforming solution as an approximation to $u$ obtained by a special postprocessing of the mixed solution.

This equivalence is exploited in $\S 8$ to derive optimal-order multigrid algorithms for the mixed and nonconforming methods. Unlike the multigrid algorithm imposed in [4] for the lowest-order Raviart-Thomas mixed triangular finite element method, our multigrid algorithms are based on standard nonconforming finite element methods. The bubble functions can be handled separately in the computations because of the orthogonality; in fact, the mixed method solution can be obtained without the need to obtain multigrid approximations to the bubble functions. The convergence of the multigrid algorithms is shown in the appendix.

The above results will be shown explicitly in two space dimensions. We will extend them to the three-dimensional case of mixed methods defined over rectangular parallelepipeds in $\S 9$, and also in an analogous way to simplices and prisms in $\S \S 10$ and 11.

Problem (1.1) arises in many practical applications. We note only that the simple formula (6.5) given below for the calculation of the flux variable $\sigma$ is very useful in calculations and in obtaining a priori estimates for the numerical electric fields of semiconductor devices [12]. 


\section{EQUIVALENT PROJECTION FINITE ELEMENT METHODS}

To define a finite element method, we need a partition $\mathscr{E}_{h}$ of $\Omega$ into elements $E$, say, simplexes, rectangular parallelepipeds, and/or prisms, where only edges or faces on $\partial \Omega$ may be curved. In $\mathscr{E}_{h}$, we also need that adjacent elements completely share their common edge or face; let $\partial \mathscr{E}_{h}$ denote the set of all interior edges $(n=2)$ or faces $(n=3) e$ of $\mathscr{E}_{h}$. We tacitly assume that $\partial \mathscr{E}_{h} \neq \varnothing$. Finally, each exterior edge or face has imposed on it either Dirichlet or Neumann conditions, but not both.

Let $V_{h} \times W_{h} \subset V \times W$ denote some standard mixed finite element space for second-order elliptic problems defined over $\mathscr{E}_{h}$ such that $\nabla \cdot V_{h}=W_{h}$ (see, e.g., $[6,7,8,13,19$, and 21]). This space is finite-dimensional and defined locally on each element $E \in \mathscr{E}_{h}$, so let $V_{h}(E)=\left.V_{h}\right|_{E}$ and $W_{h}(E)=\left.W_{h}\right|_{E}$. The constraint $V_{h} \subset V$ says that the normal components of the members of $V_{h}$ are continuous across the interior boundaries in $\partial \mathscr{E}_{h}$. Following [1], we relax this constraint on $V_{h}$ by defining

$$
\tilde{V}_{h}=\left\{v \in L^{2}(\Omega):\left.v\right|_{E} \in V_{h}(E) \text { for each } E \in \mathscr{E}_{h}\right\} .
$$

We then need to introduce Lagrange multipliers to enforce the required continuity on $\tilde{V}_{h}$, so define

$$
L_{h}=\left\{\mu \in L^{2}\left(\bigcup_{e \in \partial \mathscr{E}_{h}} e\right):\left.\left.\mu\right|_{e} \in V_{h} \cdot \nu\right|_{e} \text { for each } e \in \partial \mathscr{E}_{h}\right\} .
$$

The mixed finite element solution of (1.3) is $\left(\sigma_{h}, u_{h}\right) \in V_{h} \times W_{h}$ satisfying (2.1a) $\left(\nabla \cdot \sigma_{h}, w\right)+\left(d u_{h}, w\right)=(f, w), \quad \forall w \in W_{h}$, (2.1b) $\left(a^{-1} \sigma_{h}, v\right)-\left(u_{h}, \nabla \cdot v\right)+\left(b u_{h}, v\right)=(c, v)+(g, v \cdot \nu)_{\Gamma_{1}}, \quad \forall v \in V_{h}$.

It has a unique solution by (1.2). The unconstrained problem is to find $\left(\sigma_{h}, u_{h}, \lambda_{h}\right) \in \tilde{V}_{h} \times W_{h} \times L_{h}$ such that

$$
\begin{aligned}
& \sum_{E \in \mathscr{E}_{h}}\left(\nabla \cdot \sigma_{h}, w\right)_{E}+\left(d u_{h}, w\right)=(f, w), \quad \forall w \in W_{h}, \\
& \left(a^{-1} \sigma_{h}, v\right)-\sum_{E \in \mathscr{E}_{h}}\left[\left(u_{h}, \nabla \cdot v\right)_{E}-\left(\lambda_{h}, v \cdot \nu_{E}\right)_{\partial E \backslash \partial \Omega}\right]+\left(b u_{h}, v\right) \\
& \quad=(c, v)+(g, v \cdot \nu)_{\Gamma_{1}}, \quad \forall v \in \tilde{V}_{h}, \\
& \sum_{E \in \mathscr{E}_{h}}\left(\sigma_{h} \cdot \nu_{E}, \mu\right)_{\partial E \backslash \partial \Omega}=0, \quad \forall \mu \in L_{h} .
\end{aligned}
$$

Note that $\sigma_{h}$ and $u_{h}$ are identical in the two formulations, since (2.2c) enforces $\sigma_{h} \in V_{h}$.

We need some projection operators. Let $\mathscr{P}_{W_{h}}: L^{2}(\Omega) \rightarrow W_{h}$ denote $L^{2}(\Omega)$ projection: For $\varphi \in L^{2}(\Omega)$,

$$
\left(\varphi-\mathscr{P}_{W_{h}} \varphi, w\right)=0, \quad \forall w \in W_{h} .
$$

Similarly let $\mathscr{P}_{L_{h}}: L^{2}\left(\bigcup_{e \in \partial \varepsilon_{h}} e\right) \rightarrow L_{h}$ be $L^{2}\left(\bigcup_{e \in \partial \mathscr{C}_{h}} e\right)$-projection. To handle variable $a(x)$, we introduce the weighted $\left(L^{2}(\Omega)\right)^{n}$-projection $\tilde{\mathscr{P}}_{V_{h}}:\left(L^{2}(\Omega)\right)^{n}$ $\rightarrow \tilde{V}_{h}$ defined by

$$
\left(a^{-1}\left(\varphi-\tilde{\mathscr{P}}_{V_{h}} \varphi\right), v\right)=0, \quad \forall v \in \tilde{V}_{h}
$$


Note that each of these operators is defined locally on each $E \in \mathscr{E}_{h}$ or on each $e \in \partial \mathscr{E}_{h}$, since only $V_{h}$ has a continuity constraint.

We define now in an abstract sense our projection finite element method. Let $M_{h}$ denote some as yet unspecified finite-dimensional finite element space defined over $\mathscr{E}_{h}$ such that the degrees of freedom of $\left.M_{h}\right|_{\Gamma_{1}}$ vanish. We seek $\psi_{h} \in M_{h}-g$ satisfying

$$
\begin{aligned}
& \sum_{E \in \mathscr{E}_{h}}\left(\tilde{\mathscr{P}}_{V_{h}}\left[a\left(\nabla \psi_{h}+b \mathscr{P}_{W_{h}} \psi_{h}-c\right)\right], \nabla \xi\right)_{E}+\left(d \mathscr{P}_{W_{h}} \psi_{h}, \mathscr{P}_{W_{h}} \xi\right) \\
& \quad=\left(f, \mathscr{P}_{W_{h}} \xi\right), \quad \forall \xi \in M_{h} .
\end{aligned}
$$

Our goal is to define $M_{h}$ so that

$$
\begin{aligned}
& \sigma_{h}=-\tilde{\mathscr{P}}_{V_{h}}\left[a\left(\nabla \psi_{h}+b \mathscr{P}_{W_{h}} \psi_{h}-c\right)\right], \\
& u_{h}=\mathscr{P}_{W_{h}} \psi_{h}, \\
& \lambda_{h}=\mathscr{P}_{L_{h}} \psi_{h} .
\end{aligned}
$$

The first requirement is that $M_{h}$ give rise to a legitimate finite element method defined by (2.5); hence, we require that there exists a unique solution to the problem. Since $(2.5)$ is a square linear system, uniqueness implies existence. For uniqueness, if $\psi_{h} \in M_{h}$ satisfies

$$
\sum_{E \in \mathscr{E}_{h}}\left(\tilde{\mathscr{P}}_{V_{h}}\left[a\left(\nabla \psi_{h}+b \mathscr{P}_{W_{h}} \psi_{h}\right)\right], \nabla \xi\right)_{E}+\left(d \mathscr{P}_{W_{h}} \psi_{h}, \mathscr{P}_{W_{h}} \xi\right)=0, \quad \forall \xi \in M_{h},
$$

then we need to show that $\psi_{h}=0$. Take $\xi=\psi_{h}$, note that by (2.4),

$$
\begin{aligned}
\left(\tilde{\mathscr{P}}_{V_{h}}\left(a \nabla \psi_{h}\right), \nabla \psi_{h}\right)_{E} & =\left(a^{-1} \tilde{\mathscr{P}}_{V_{h}}\left(a \nabla \psi_{h}\right), a \nabla \psi_{h}\right)_{E} \\
& =\left(a^{-1} \tilde{\mathscr{P}}_{V_{h}}\left(a \nabla \psi_{h}\right), \tilde{\mathscr{P}}_{V_{h}}\left(a \nabla \psi_{h}\right)\right)_{E}, \\
\left(\tilde{\mathscr{P}}_{V_{h}}\left(a b \mathscr{P}_{W_{h}} \psi_{h}\right), \nabla \psi_{h}\right)_{E} & =\left(a^{-1} \tilde{\mathscr{P}}_{V_{h}}\left(a b \mathscr{P}_{W_{h}} \psi_{h}\right), a \nabla \psi_{h}\right)_{E} \\
& =\left(b \mathscr{P}_{W_{h}} \psi_{h}, \tilde{\mathscr{P}}_{V_{h}}\left(a \nabla \psi_{h}\right)\right)_{E},
\end{aligned}
$$

and then apply coercivity (1.2) to conclude that both $\left\|\tilde{\mathscr{P}}_{V_{h}}\left(a \nabla \psi_{h}\right)\right\|_{\left(L^{2}(\Omega)\right)^{n}}=0$ and $\left(d \mathscr{P}_{W_{h}} \psi_{h}, \mathscr{P}_{W_{h}} \psi_{h}\right)=0$. The former requires that the $\tilde{\mathscr{P}}_{V_{h}}$-projection of $a \nabla \psi_{h}$ be zero on each $E \in \mathscr{E}_{h}$ :

$$
\left(a^{-1} a \nabla \psi_{h}, v\right)_{E}=0, \quad \forall v \in V_{h}(E) .
$$

We therefore require of the space $M_{h}$ the first condition:

(C1) For $\xi \in M_{h}$, if $(\nabla \xi, v)_{E}=0$ for all $v \in V_{h}(E)$ and all $E \in \mathscr{E}_{h}$, and if $\left(d \mathscr{P}_{W_{h}} \xi, \mathscr{P}_{W_{h}} \xi\right)=0$, then $\xi=0$.

In order that $(2.6 \mathrm{c})$ makes sense, we require that

(C2) For $\xi \in M_{h}$, its projection $\mathscr{P}_{L_{h}} \xi$ can be uniquely defined on each $e \in \partial \mathscr{E}_{h}$.

We can consider now the equivalence of the two schemes (2.2) and (2.5). It is convenient to take $\psi_{h}$ as given by (2.5) and let $\sigma_{h}, u_{h}$, and $\lambda_{h}$ be given by (2.6). We then show that $(2.2)$ results. 
By the definitions (2.6), definitions (2.3) and (2.4), and finally integration by parts, we see that for any $v \in \tilde{V}_{h}$,

$$
\begin{aligned}
\left(a^{-1}\right. & \left.\sigma_{h}, v\right)-\sum_{E \in \mathscr{E}_{h}}\left[\left(u_{h}, \nabla \cdot v\right)_{E}-\left(\lambda_{h}, v \cdot \nu_{E}\right)_{\partial E \backslash \partial \Omega}\right]+\left(b u_{h}, v\right) \\
= & -\left(a^{-1} \tilde{\mathscr{P}}_{V_{h}}\left[a\left(\nabla \psi_{h}+b \mathscr{P}_{W_{h}} \psi_{h}-c\right)\right], v\right) \\
& -\sum_{E \in \mathscr{E}_{h}}\left[\left(\mathscr{P}_{W_{h}} \psi_{h}, \nabla \cdot v\right)_{E}-\left(\mathscr{P}_{L_{h}} \psi_{h}, v \cdot \nu_{E}\right)_{\partial E \backslash \partial \Omega}\right]+\left(b \mathscr{P}_{W_{h}} \psi_{h}, v\right) \\
= & -\sum_{E \in \mathscr{E}_{h}}\left(\nabla \psi_{h}+b \mathscr{P}_{W_{h}} \psi_{h}-c, v\right)_{E} \\
& -\sum_{E \in \mathscr{E}_{h}}\left[\left(\psi_{h}, \nabla \cdot v\right)_{E}-\left(\psi_{h}, v \cdot \nu_{E}\right)_{\partial E \backslash \partial \Omega}\right]+\left(b \mathscr{P}_{W_{h}} \psi_{h}, v\right) \\
= & \sum_{E \in \mathscr{E}_{h}}\left[-\left(\nabla \psi_{h}-c, v\right)_{E}+\left(\nabla \psi_{h}, v\right)_{E}\right]+(g, v \cdot \nu)_{\Gamma_{1}} \\
= & (c, v)+(g, v \cdot \nu)_{\Gamma_{1}}
\end{aligned}
$$

this is $(2.2 b)$.

For (2.2a)and (2.2c), we integrate the first term on the left-hand side of (2.5) by parts to see that for any $\xi \in M_{h}$,

$$
\sum_{E \in \mathscr{E}_{h}}\left(\tilde{\mathscr{P}}_{V_{h}}\left[a\left(\nabla \psi_{h}+b \mathscr{P}_{W_{h}} \psi_{h}-c\right)\right], \nabla \xi\right)_{E}=\sum_{E \in \mathscr{E}_{h}}\left[\left(\nabla \cdot \sigma_{h}, \xi\right)_{E}-\left(\sigma_{h} \cdot \nu_{E}, \xi\right)_{\partial E}\right] ;
$$

hence, introducing two projection operators, (2.5) becomes

$$
\begin{aligned}
& \sum_{E \in \mathscr{E}_{h}}\left(\nabla \cdot \sigma_{h}, \mathscr{P}_{W_{h}} \xi\right)_{E}+\left(d u_{h}, \mathscr{P}_{W_{h}} \xi\right)-\sum_{E \in \mathscr{E}_{h}}\left(\sigma_{h} \cdot \nu_{E}, \mathscr{P}_{L_{h}} \xi\right)_{\partial E \backslash \partial \Omega} \\
& \quad=\left(f, \mathscr{P}_{W_{h}} \xi\right), \quad \forall \xi \in M_{h},
\end{aligned}
$$

where $\mathscr{P}_{L_{h}} \xi$ on $\partial E$ is defined on the trace of $\xi$ from within $E$. To separate information on $\partial E$ from that in $E$, we require the third condition on $M_{h}$ :

(C3) For any $(w, \mu) \in W_{h} \times L_{h}$, there exist $\xi_{1}, \xi_{2} \in M_{h}$ such that

$$
\text { (i) }\left\{\begin{array} { l } 
{ \mathscr { P } _ { W _ { h } } \xi _ { 1 } = w , } \\
{ \mathscr { P } _ { L _ { h } } \xi _ { 1 } = 0 }
\end{array} \quad \text { and } \quad \text { (ii) } \left\{\begin{array}{l}
\mathscr{P}_{W_{h}} \xi_{2}=0 \\
\mathscr{P}_{L_{h}} \xi_{2}=\mu
\end{array}\right.\right.
$$

The $\xi_{1}$ gives us $(2.2 \mathrm{a})$ while the $\xi_{2}$ gives us $(2.2 \mathrm{c})$.

Since any $u_{h}$ and $\lambda_{h}$ can arise as a solution to (2.2) by adjusting the data, condition (C3) is also necessary for the equivalence. We have shown the following theorem.

Theorem 1. For a given mixed finite element method (2.1) or (2.2) such that $W_{h}=\nabla \cdot V_{h}$, the projection finite element method (2.5) is well defined if, and only if, $M_{h}$ satisfies (C1). Moreover, if $M_{h}$ satisfies (C1) and (C2), these two methods are equivalent by the relations (2.6) if, and only if, $M_{h}$ satisfies (C3).

Theorem 2. If a given projection finite element method (2.5) with projection space $V_{h}$ (and $W_{h}=\nabla \cdot V_{h}$ and $L_{h}$ defined from $V_{h}$ ) satisfies (C1)-(C3) and the property that for any $\xi \in M_{h}$ such that $\mathscr{P}_{L_{h}} \xi=0$,

$$
\sup _{v \in V_{h} \backslash\{0\}} \frac{\sum_{E \in \mathscr{E}_{h}}(v, \nabla \xi)_{E}}{\|v\|_{\left(L^{2}(\Omega)\right)^{n}}} \geq \kappa_{h}\left\|\mathscr{P}_{W_{h}} \xi\right\|_{L^{2}(\Omega)}
$$


for some $\kappa_{h}>0$, then $V_{h}$ gives rise to an equivalent mixed method (2.1) or (2.2) for which $V_{h}$ and $W_{h}$ satisfy the inf-sup condition [5] for the constant $\kappa_{h}$ : For any $w \in W_{h}$,

$$
\sup _{v \in V_{h} \backslash\{0\}} \frac{(\nabla \cdot v, w)}{\|v\|_{\left(L^{2}(\Omega)\right)^{n}}} \geq \kappa_{h}\|w\|_{L^{2}(\Omega)} .
$$

Moreover, if (2.10) holds uniformly in $h$, i.e., $\kappa_{h}=\kappa$ is independent of $h$, then also the inf-sup condition holds uniformly in $h$.

Proof. For $w \in W_{h}$, we can choose by (C3) $\xi \in M_{h}$ such that $\mathscr{P}_{W_{h}} \xi=-w$ and $\mathscr{P}_{L_{h}} \xi=0$. For this $\xi,(2.10)$ is the inf-sup condition after an integration by parts.

\section{ON THE LOCAL CONSTRUCTION OF $M_{h}$}

It is not yet clear whether an appropriate $M_{h}$ can be constructed for a given mixed method. In this section we consider the question of how to construct such an $M_{h}$. We do not discuss problems associated with the outer boundary of the domain, but instead concentrate on the local spaces defined on some $E \in \mathscr{E}_{h}$ with edges or faces $e \in \partial \mathscr{E}_{h}$.

We begin by noting that dimensional considerations for satisfying $(\mathrm{Cl})$ and (C3) easily show the following corollary of Theorem 1 , wherein $M_{h}(E)=\left.M_{h}\right|_{E}$ and $L_{h}(e)=\left.L_{h}\right|_{e}$.

Corollary 1. If a given mixed finite element method (2.1) or (2.2) (with $W_{h}=$ $\left.\nabla \cdot V_{h}\right)$ is equivalent to the projection finite element method (2.5) by the relations (2.6), then, for each $E \in \mathscr{E}_{h}$ such that $\partial E \cap \partial \Omega=\varnothing$,

$$
\operatorname{dim}\left(W_{h}(E)\right)+\sum_{e \subset \partial E} \operatorname{dim}\left(L_{h}(e)\right) \leq \operatorname{dim}\left(M_{h}(E)\right) \leq \operatorname{dim}\left(V_{h}(E)\right)+1 .
$$

The left-hand side of the inequality follows from (C3), and the right-hand side from $(\mathrm{C} 1)$. This result can be used to bound the dimension of $M_{h}(E)$; it may even show that $M_{h}(E)$ cannot exist for some novel mixed methods.

We now localize the condition $(\mathrm{C} 1)$ as follows:

$\left(\mathrm{C1}^{\prime}\right)$ For $\xi \in M_{h}(E)$, if $(\nabla \xi, v)_{E}=0$ for all $v \in V_{h}(E)$, then $\xi$ is constant on $E$.

Theorem 3. Suppose that $V_{h} \times W_{h}$ is a mixed finite element space such that $W_{h}=\nabla \cdot V_{h}, 1 \in W_{h}(E)$ for each $E \in \mathscr{E}_{h}$, and $1 \in L_{h}(e)$ for each $e \in \partial \mathscr{E}_{h}$. If $M_{h}$ satisfies $\left(\mathrm{C1}^{\prime}\right)$ for each $E \in \mathscr{E}_{h}$ and $(\mathrm{C} 2)$, then $M_{h}$ satisfies $(\mathrm{C} 1)$.

Proof. For some $\xi \in M_{h}$, suppose that $(\nabla \xi, v)_{E}=0$ for all $v \in V_{h}(E)$ and $E \in \mathscr{E}_{h}$, and $\left(d \mathscr{P}_{W_{h}} \xi, \mathscr{P}_{W_{h}} \xi\right)=0$. We conclude from $\left(\mathrm{Cl}^{\prime}\right)$ that $\xi$ is constant on each $E$. Since $(C 2)$ requires a unique definition of $\mathscr{P}_{L_{h}} \xi$, in fact $\xi$ is a constant on all of $\Omega$. Finally, either $\Gamma_{1} \neq \varnothing$ or $d>0$ implies that $\xi=0$.

The mixed method spaces that we consider have the property that there exists a projection operator $\Pi_{h}:\left(H^{1}(E)\right)^{n} \rightarrow V_{h}(E)$ such that

$$
\begin{aligned}
& \nabla \cdot\left(\Pi_{h} v\right)=\mathscr{P}_{W_{h}}(\nabla \cdot v), \\
& \left(\Pi_{h} v\right) \cdot \nu=\mathscr{P}_{L_{h}}(v \cdot \nu) .
\end{aligned}
$$

We exploit this fact in the following way. 
Theorem 4. Suppose that $E$ is convex and that $V_{h}(E) \times W_{h}(E)$ is a mixed finite element space such that $W_{h}(E)=\nabla \cdot V_{h}(E), 1 \in W_{h}(E), 1 \in L_{h}(e)$ for each $e \subset \partial E$, and there exists an operator $\Pi_{h}:\left(H^{1}(E)\right)^{n} \rightarrow V_{h}(E)$ satisfying (3.1). If $M_{h}(E)$ is a space of functions such that

$$
\operatorname{dim}\left(M_{h}(E)\right)=\operatorname{dim}\left(W_{h}(E)\right)+\sum_{e \subset \partial E} \operatorname{dim}\left(L_{h}(e)\right)
$$

with unisolvent degrees of freedom described by

(DF1) $(\xi, w)_{E}$ for all $w$ in a basis of $W_{h}(E)$,

(DF2) $(\xi, \mu)_{e}$ for all $\mu$ in a basis of $L_{h}(e)$, for each $e \subset \partial E$,

and if $M_{h}(E)$ contains the constant functions, then $M_{h}(E)$ satisfies $\left(\mathrm{C1}^{\prime}\right),(\mathrm{C} 2)$, and $(\mathrm{C} 3)$.

Proof. The hypotheses (DF) give (C2) and (C3), so we need only show (C1' ). Let $A_{S}(\varphi)=(\varphi, 1)_{S} /(1,1)_{E}$ denote a type of average of a function $\varphi(x)$ on $S \subset E$. For $\xi \in M_{h}(E)$, if $\zeta=\xi-A_{E}(\xi)$ and

$$
(\nabla \xi, v)_{E}=(\nabla \zeta, v)_{E}=-(\zeta, \nabla \cdot v)_{E}+\sum_{e \subset \partial E}(\zeta, v \cdot \nu)_{e}=0
$$

for all $v \in V_{h}(E)$, then we need to show that $\zeta=0$.

Given any $w \in W_{h}$, there is some $\tilde{v} \in V_{h}$ such that $\nabla \cdot \tilde{v}=w$. Solve the problem

$$
\begin{aligned}
& \Delta \varphi=A_{\partial E}(\tilde{v} \cdot \nu) \quad \text { in } E, \\
& \nabla \varphi \cdot \nu=\tilde{v} \cdot \nu \quad \text { on } \partial E
\end{aligned}
$$

and set $v=\tilde{v}-\Pi_{h} \nabla \varphi \in V_{h}$. Then (3.1) implies that $v \cdot \nu=0$ on $\partial E$ and $\nabla \cdot v=w-A_{\partial E}(\tilde{v} \cdot \nu)$. As a consequence, (3.2) implies that $\mathscr{P}_{W_{h}} \zeta=0$.

Now for $e \subset \partial E$, take any $\lambda \in L_{h}(e)$ and then any $\tilde{v} \in V_{h}$ such that $\tilde{v} \cdot \nu=\lambda$ on $e$. Solve the problem

$$
\begin{aligned}
& \Delta \varphi=\nabla \cdot \tilde{v}-A_{E}(\nabla \cdot \tilde{v})+A_{\partial E \backslash e}(\tilde{v} \cdot \nu) \text { in } E, \\
& \nabla \varphi \cdot \nu=\tilde{v} \cdot \nu \text { on } \partial E \backslash e, \\
& \nabla \varphi \cdot \nu=0 \text { on } e,
\end{aligned}
$$

and again set $v=\tilde{v}-\Pi_{h} \nabla \varphi \in V_{h}$. Then (3.1) and (3.2) imply that $\mathscr{P}_{L_{h}} \zeta=0$ on $e$.

By the unisolvence of the degrees of freedom, since $\zeta \in M_{h}$, we conclude that $\zeta=0$.

\section{EQUIVALENT SPACES FOR TRIANGULAR MIXED METHODS}

We are now in a position to construct some nonconforming spaces that give rise to projection finite element methods that are equivalent to standard mixed methods. We begin by generalizing the results of Arnold and Brezzi [1] to the known triangular methods. These mixed spaces satisfy the conditions of Theorem 4 , so it remains only to define over a triangle $T$ a space $M_{h}(T)$ of the correct dimension and prove the unisolvence of (DF).

Let $P_{k}(E)$ denote the space of polynomials of total degree less than or equal to $k$ defined in $E$. We will make use of the barycentric coordinates $\hat{\ell}_{i}, i=$ 
$1,2,3$, defined on $T$ to be the unique affine functions that take the value one at vertex $i$, and the value zero on the opposite edge. Finally, for any edge $e$, let $\tilde{P}_{k}(e)$ denote the $L^{2}(e)$-orthogonal complement of $P_{k}(e)$ in $P_{k-1}(e)$ (i.e., the span of the Legendre polynomials of exact degree $k$ ).

4.1. The Raviart-Thomas spaces on triangles. These spaces [21] are defined for each $k \geq 0$ by

$$
\begin{aligned}
& V_{h}^{k}(T)=\left(P_{k}(T)\right)^{2} \oplus\left((x, y) P_{k}(T)\right), \\
& W_{h}^{k}(T)=P_{k}(T), \\
& L_{h}^{k}(e)=P_{k}(e) .
\end{aligned}
$$

First let us recall what is already known for the lowest-order space. An $M_{h}$ (of dimension 4) for this space is $[1,11]$

$$
M_{h}(T)=P_{1}(T) \oplus B_{h}(T),
$$

where we define $B_{h}(T)$ to be the span of either the $P_{3}$-bubble function,

$$
\beta_{T}^{3}(x, y)=\hat{\ell}_{1}(x, y) \hat{\ell}_{2}(x, y) \hat{\ell}_{3}(x, y),
$$

which vanishes on each edge, or the $P_{2}$-bubble function,

$$
\beta_{T}^{2}(x, y)=2-3\left(\hat{\ell}_{1}^{2}(x, y)+\hat{\ell}_{2}^{2}(x, y)+\hat{\ell}_{3}^{2}(x, y)\right),
$$

which vanishes at the two quadratic Gauss points on each edge.

For $\xi \in M_{h}$, we can write $\xi=\xi_{1}+\xi_{2}$ for $\xi_{1} \in P_{1}(T)$ and $\xi_{2} \in B_{h}(T)$, and then the degrees of freedom for the element are normally given as the value of:

(i) $\int_{T} \xi(x) d x$;

(ii) $\xi_{1}$ at the midpoint of each edge $e \subset \partial T$.

(Note that if $B_{h}(T)=\operatorname{span}\left\{\beta_{T}^{3}\right\}$, we may replace $\xi_{1}$ by $\xi$ in (ii).) An equivalent set of degrees of freedom can be given by the value of (i) and

(ii' ) $\int_{e} \xi(x) d \sigma(x)$ for each edge $e \subset \partial T$;

(ii) and (ii' ${ }^{\prime}$ ) are equivalent since midpoint quadrature is exact for linear functions. These degrees of freedom are (DF1) and (DF2), and their unisolvence is known.

For the family as a whole, we define

$$
M_{h}^{k}(T)= \begin{cases}\left\{v \in P_{k+3}(T): v \mid e \in P_{k+1}(e)\right\} & \text { if } k \text { is even, } \\ \left\{v \in P_{k+3}(T): v \mid e \in P_{k}(e) \oplus \tilde{P}_{k+2}(e)\right\} & \text { if } k \text { is odd. }\end{cases}
$$

We first show that $M_{h}^{k}(T)$ has the correct dimension. The dimension of $P_{k+3}(T)$ is $\frac{1}{2}(k+5)(k+4)$, which is exactly six more than $\operatorname{dim}\left(W_{h}(T)\right)+$ $3 \operatorname{dim}\left(L_{h}(e)\right)=\frac{1}{2}(k+8)(k+1)$. For simplicity, assume that $k$ is even; the odd case is similar. For any $\xi \in P_{k+3}(T)$, we can write that

$$
\xi(x)=\sum_{0 \leq i+j \leq k+3} a_{i, j} \hat{\ell}_{1}^{i}(x) \hat{\ell}_{2}^{j}(x)
$$


for some constants $a_{i, j}$. If now $\xi \in M_{h}^{k}(T)$, then $\left.\xi\right|_{e_{1}} \in P_{k+1}\left(e_{1}\right)$ implies that $a_{0, k+3}=a_{0, k+2}=0$, and $\left.\xi\right|_{e_{2}} \in P_{k+1}\left(e_{2}\right)$ impiies that $a_{k+3,0}=a_{k+2,0}=0$. On $e_{3}, \hat{\ell}_{2}=1-\hat{\ell}_{1}$, so

$$
\left.\xi\right|_{e_{3}}=\sum_{0 \leq i+j \leq k+3} a_{i, j} \hat{\ell}_{1}^{i}\left(1-\hat{\ell}_{1}\right)^{j} \in P_{k+1}\left(e_{3}\right)
$$

implies that

$$
\sum_{i+j=k+3}(-1)^{j} a_{i, j}=0 \text { and } \sum_{i+j=k+2}(-1)^{j} a_{i, j}+\sum_{i+j=k+3} j(-1)^{j-1} a_{i, j}=0 .
$$

These six conditions are clearly independent, so $M_{h}^{k}(T)$ has the correct dimension.

Now we consider the unisolvence of (DF). Suppose that $\xi \in M_{h}^{k}(T)$ has degrees of freedom (DF) equal to zero. The (DF2) imply that on each edge $e, \xi$ is a Legendre polynomial of degree $k+1$ if $k$ is even and $k+2$ if $k$ is odd, i.e., of odd degree. Since the odd-degree Legendre polynomials are odd functions, traversing $\partial T$, we see that $\xi$ must vanish identically on the boundary. As a consequence, we write that $\xi=\hat{\ell}_{1} \hat{\ell}_{2} \hat{\ell}_{3} w$ for some $w \in P_{k}(T)$. Now (DF1) shows that $\left(\hat{\ell}_{1} \hat{\ell}_{2} \hat{\ell}_{3} w, w\right)_{T}=0$, which finally gives that $\xi=0$.

We remark that if $k$ is even, we obtain the nonconforming method of Arnold and Brezzi [1].

4.2. The Brezzi-Douglas-Marini spaces on triangles. These spaces [8] can be defined for each $k \geq 1$ by

$$
\begin{aligned}
& V_{h}^{k}(T)=\left(P_{k}(T)\right)^{2}, \\
& W_{h}^{k}(T)=P_{k-1}(T), \\
& L_{h}^{k}(e)=P_{k}(e) .
\end{aligned}
$$

Let us define

$$
M_{h}^{k}(T)= \begin{cases}\left\{v \in P_{k+2}(T): v \mid e \in P_{k+1}(e)\right\} & \text { if } k \text { is even } \\ \left\{v \in P_{k+2}(T): v \mid e \in P_{k}(e) \oplus \tilde{P}_{k+2}(e)\right\} & \text { if } k \text { is odd }\end{cases}
$$

Since $\operatorname{dim}\left(P_{k+2}(T)\right)=\frac{1}{2}(k+4)(k+3)$ is exactly three more than $\operatorname{dim}\left(W_{h}(T)\right)+$ $3 \operatorname{dim}\left(L_{h}(e)\right)=\frac{1}{2}(k+6)(k+1)$, an argument as above shows that $M_{h}^{k}(T)$ has the correct dimension. The unisolvence of (DF) is also shown as above.

\section{EQUiVALENT SPACES FOR RECTANGULAR PARALLELEPIPED MIXED METHODS}

We now construct some nonconforming spaces that give rise to projection finite element methods that are equivalent to standard mixed methods defined over a rectangle or rectangular parallelepiped $R \subset \mathbb{R}^{n}, n=2$ or 3 . Again the mixed spaces satisfy the conditions of Theorem 4 .

For simplicity, assume that $R=[-1,1]^{n}$. We will make use of the Legendre polynomials $p_{m}\left(x_{i}\right)$ of degree $m$ defined on the interval $[-1,1]$. Recall that $P_{k}(R)$ is the space of polynomials of total degree less than or equal to $k$ defined in $R$, and let $Q_{k, \ell, m}(R)$ denote the space of polynomials of degree less than or equal to $k$ in $x_{1}, \ell$ in $x_{2}$, and $m$ in $x_{3}$ (where $m$ and $x_{3}$ are absent if $n=2$ ). 
5.1. The Raviart-Thomas spaces on rectangles. These spaces [21] are defined for each $k \geq 0$ by

$$
\begin{aligned}
& V_{h}^{k}(R)=Q_{k+1, k}(R) \times Q_{k, k+1}(R), \\
& W_{h}^{k}(R)=Q_{k, k}(R), \\
& L_{h}^{k}(e)=P_{k}(e) .
\end{aligned}
$$

We define

$$
M_{h}^{k}(R)=Q_{k+2, k}(R) \oplus Q_{k, k+2}(R)=Q_{k, k}(R) \oplus A^{k}(R) \oplus B^{k}(R),
$$

where

$$
\begin{aligned}
& A^{k}(R)=\left\{\sum_{i=0}^{k}\left[a_{i, 1} p_{k+1}\left(x_{1}\right)+a_{i, 2} p_{k+2}\left(x_{1}\right)\right] p_{i}\left(x_{2}\right): a_{i, j} \in \mathbb{R}\right\}, \\
& B^{k}(R)=\left\{\sum_{i=0}^{k} p_{i}\left(x_{1}\right)\left[b_{i, 1} p_{k+1}\left(x_{2}\right)+b_{i, 2} p_{k+2}\left(x_{2}\right)\right]: b_{i, j} \in \mathbb{R}\right\} .
\end{aligned}
$$

Note that $\operatorname{dim}\left(A^{k}(R)\right)=\operatorname{dim}\left(B^{k}(R)\right)=2(k+1)$, so it is trivial to verify that $\operatorname{dim}\left(M_{h}^{k}(R)\right)=\operatorname{dim}\left(W_{h}^{k}(R)\right)+4 \operatorname{dim}\left(L_{h}^{k}(e)\right)$.

We need to show that the degrees of freedom (DF) are independent. Assume that the (DF) are zero for some $\xi \in M_{h}^{k}(R)=\xi_{1}+\xi_{2}+\xi_{3}$, where $\xi_{1} \in Q_{k, k}(R)$, $\xi_{2} \in A^{k}(R)$, and $\xi_{3} \in B^{k}(R)$. By the orthogonality of the Legendre polynomials, (DF1) is zero for $A^{k}(R)$ and $B^{k}(R)$, so (DF1) implies that $\xi_{1}=0$. On the two sides where $x_{1}= \pm 1,(\mathrm{DF} 2)$ for $B^{k}(R)$ is zero, but for $A^{k}(R)$ we have

$$
\sum_{i=0}^{k} \int_{-1}^{1}\left[a_{i, 1} p_{k+1}( \pm 1)+a_{i, 2} p_{k+2}( \pm 1)\right] p_{i}\left(x_{2}\right) \varphi\left(x_{2}\right) d x_{2}=0, \quad \forall \varphi \in P_{k}([-1,1])
$$

and so $a_{i, 1} p_{k+1}( \pm 1)+a_{i, 2} p_{k+2}( \pm 1)=0$ for each $i$. Since the Legendre polynomials are alternately even and odd, we conclude that $a_{i, 1}=a_{i, 2}=0$ for each $i$, i.e., $\xi_{2}=0$. Similarly, on the sides where $x_{2}= \pm 1$, we conclude that $\xi_{3}=0$, and so $\xi=0$ and we have our unisolvence.

We omit the proofs of unisolvence below, since they are similar to that given above.

5.2. The Brezzi-Douglas-Marini spaces on rectangles. These spaces [8] are defined for each $k \geq 1$ as

$$
\begin{aligned}
& V_{h}^{k}(R)=\left(P_{k}(R)\right)^{2} \oplus \operatorname{span}\left\{\operatorname{curl} x_{1}^{k+1} x_{2}, \operatorname{curl} x_{1} x_{2}^{k+1}\right\}, \\
& W_{h}^{k}(R)=P_{k-1}(R), \\
& L_{h}^{k}(e)=P_{k}(e),
\end{aligned}
$$

where curl $w=\left(-\partial w / \partial x_{2}, \partial w / \partial x_{1}\right)$. We define

$$
M_{h}^{k}(R)=P_{k-1}(R) \oplus A^{k}(R) \oplus B^{k}(R),
$$

where $A^{k}(R)$ and $B^{k}(R)$ are defined above by (5.1). 
5.3. The Brezzi-Douglas-Fortin-Marini spaces on rectangles. Also called reduced Brezzi-Douglas-Marini spaces [7], they can be defined for each $k \geq 0$ as

$$
\begin{aligned}
& V_{h}^{k}(R)=\left\{\varphi \in P_{k+1}(R): \text { the coefficient of } x_{2}^{k+1} \text { vanishes }\right\} \\
& \times\left\{\varphi \in P_{k+1}(R): \text { the coefficient of } x_{1}^{k+1} \text { vanishes }\right\}, \\
& W_{h}^{k}(R)=P_{k}(R) \text {, } \\
& L_{h}^{k}(e)=P_{k}(e) \text {. }
\end{aligned}
$$

Now we define

$$
M_{h}^{k}(R)=P_{k}(R) \oplus A^{k}(R) \oplus B^{k}(R) .
$$

Again, $A^{k}(R)$ and $B^{k}(R)$ are defined by $(5.1)$.

5.4. The Raviart-Thomas-Nedelec spaces on rectangular parallelepipeds. These spaces are the three-dimensional analogues of the Raviart-Thomas spaces on rectangles, and they are defined $[19,21]$ for each $k \geq 0$ by

$$
\begin{aligned}
& V_{h}^{k}(R)=Q_{k+1, k, k}(R) \times Q_{k, k+1, k}(R) \times Q_{k, k, k+1}(R), \\
& W_{h}^{k}(R)=Q_{k, k, k}(R), \\
& L_{h}^{k}(e)=Q_{k, k}(e) .
\end{aligned}
$$

We define

$$
\begin{aligned}
M_{h}^{k}(R) & =Q_{k+2, k, k}(R) \oplus Q_{k, k+2, k}(R) \oplus Q_{k, k, k+2}(R) \\
& =Q_{k, k, k}(R) \oplus A^{k}(R) \oplus B^{k}(R) \oplus C^{k}(R),
\end{aligned}
$$

where

$$
\begin{aligned}
& A^{k}(R)=\left\{\sum_{i=0}^{k} \sum_{j=0}^{k}\left[a_{i, j, 1} p_{k+1}\left(x_{1}\right)+a_{i, j, 2} p_{k+2}\left(x_{1}\right)\right] p_{i}\left(x_{2}\right) p_{j}\left(x_{3}\right): a_{i, j, \ell} \in \mathbb{R}\right\}, \\
& B^{k}(R)=\left\{\sum_{i=0}^{k} \sum_{j=0}^{k} p_{i}\left(x_{1}\right)\left[b_{i, j, 1} p_{k+1}\left(x_{2}\right)+b_{i, j, 2} p_{k+2}\left(x_{2}\right)\right] p_{j}\left(x_{3}\right): b_{i, j, \ell} \in \mathbb{R}\right\}, \\
& C^{k}(R)=\left\{\sum_{i=0}^{k} \sum_{j=0}^{k} p_{i}\left(x_{1}\right) p_{j}\left(x_{2}\right)\left[c_{i, j, 1} p_{k+1}\left(x_{3}\right)+c_{i, j, 2} p_{k+2}\left(x_{3}\right)\right]: c_{i, j, \ell} \in \mathbb{R}\right\} .
\end{aligned}
$$

5.5. The Brezzi-Douglas-Durán-Fortin spaces on rectangular parallelepipeds. These spaces [6] are the three-dimensional analogues of the Brezzi-DouglasMarini spaces on rectangles. They are defined for $k \geq 1$ by

$$
\begin{aligned}
& V_{h}^{k}(R)=\left(P_{k}(R)\right)^{3} \oplus \operatorname{span}\left\{\operatorname{curl}\left(0,0, x_{1}^{k+1} x_{2}\right), \operatorname{curl}\left(0, x_{1} x_{3}^{k+1}, 0\right),\right. \\
& \operatorname{curl}\left(x_{2}^{k+1} x_{3}, 0,0\right), \operatorname{curl}\left(0,0, x_{1} x_{2}^{i+1} x_{3}^{k-i}\right), \\
& \left.\operatorname{curl}\left(0, x_{1}^{i+1} x_{2}^{k-i} x_{3}, 0\right), \operatorname{curl}\left(x_{1}^{k-i} x_{2} x_{3}^{i+1}, 0,0\right)\right\} \text {, } \\
& W_{h}^{k}(R)=P_{k-1}(R), \\
& L_{h}^{k}(e)=P_{k}(e) \text {. }
\end{aligned}
$$

We define

$$
M_{h}^{k}(R)=P_{k-1}(R) \oplus A^{k}(R) \oplus B^{k}(R) \oplus C^{k}(R),
$$


where

$$
A^{k}(R)=\left\{\sum_{0 \leq i+j \leq k}\left[a_{i, j, 1} p_{k+1}\left(x_{1}\right)+a_{i, j, 2} p_{k+2}\left(x_{1}\right)\right] p_{i}\left(x_{2}\right) p_{j}\left(x_{3}\right): a_{i, j, \ell} \in \mathbb{R}\right\},
$$

$$
B^{k}(R)=\left\{\sum_{0 \leq i+j \leq k} p_{i}\left(x_{1}\right)\left[b_{i, j, 1} p_{k+1}\left(x_{2}\right)+b_{i, j, 2} p_{k+2}\left(x_{2}\right)\right] p_{j}\left(x_{3}\right): b_{i, j, \ell} \in \mathbb{R}\right\},
$$

$$
C^{k}(R)=\left\{\sum_{0 \leq i+j \leq k} p_{i}\left(x_{1}\right) p_{j}\left(x_{2}\right)\left[c_{i, j, 1} p_{k+1}\left(x_{3}\right)+c_{i, j, 2} p_{k+2}\left(x_{3}\right)\right]: c_{i, j, \ell} \in \mathbb{R}\right\} .
$$

5.6. The Brezzi-Douglas-Fortin-Marini spaces on rectangular parallelepipeds. These spaces [7] are also called reduced Brezzi-Douglas-Durán-Fortin spaces, and they can be defined for each $k \geq 0$ as

$$
\begin{aligned}
& V_{h}^{k}(R)=\{\varphi \in \\
& \left.\quad P_{k+1}(R): \text { the coefficient of } \sum_{i=0}^{k+1} x_{2}^{k+1-i} x_{3}^{i} \text { vanishes }\right\} \\
& \times\left\{\varphi \in P_{k+1}(R): \text { the coefficient of } \sum_{i=0}^{k+1} x_{1}^{k+1-i} x_{3}^{i} \text { vanishes }\right\} \\
& \quad \times\left\{\varphi \in P_{k+1}(R): \text { the coefficient of } \sum_{i=0}^{k+1} x_{1}^{k+1-i} x_{2}^{i} \text { vanishes }\right\}, \\
& W_{h}^{k}(R)=P_{k}(R),
\end{aligned}
$$

We define

$$
M_{h}^{k}(R)=P_{k}(R) \oplus A^{k}(R) \oplus B^{k}(R) \oplus C^{k}(R),
$$

where $A^{k}(R), B^{k}(R)$, and $C^{k}(R)$ are defined in the previous subsection by (5.2).

\section{IMPLEMENTATION OF THE LOWEST-ORDER RAVIART-THOMAS METHOD ON RECTANGLES}

We now concentrate our attention to the lowest-order Raviart-Thomas spaces over rectangles [21] (or equivalently the lowest-order Brezzi-Douglas-FortinMarini spaces [7]), since these are widely used in practice. In this and the following three sections, let $\Omega$ be a planar domain, let $\mathscr{E}_{h}$ be a family of quasiregular partitions of $\Omega$ into rectangles oriented along the coordinate axes with maximum diameter $h$, and let $a$ be diagonal. For simplicity of exposition, assume that $a$ is a scalar, $\Gamma_{2}=\varnothing$, and $g=0$.

The lowest-order Raviart-Thomas spaces [21] are

$$
\begin{aligned}
V_{h}= & \left\{v:\left.v\right|_{R}=\left(a_{R}^{1}+a_{R}^{2} x, a_{R}^{3}+a_{R}^{4} y\right), a_{R}^{i} \in \mathbb{R}, \forall R \in \mathscr{E}_{h} ;\right. \\
& \left.v \cdot n \text { is continuous at the interelement edges of } \mathscr{E}_{h}\right\}, \\
W_{h}= & \left\{w:\left.w\right|_{R} \text { is constant, } \forall R \in \mathscr{E}_{h}\right\}, \\
L_{h}= & \left\{\mu:\left.\mu\right|_{e} \text { is constant, } \forall e \in \partial \mathscr{E}_{h}\right\} .
\end{aligned}
$$


A general, equivalent, nonconforming method is defined above in $\S 5.1$ (and also in $\S 5.3$ ) for the space

$$
\begin{aligned}
& M_{h}=\left\{\xi:\left.\xi\right|_{R}=a_{R}^{1}+a_{R}^{2} x+a_{R}^{3} y+a_{R}^{4} x^{2}+a_{R}^{5} y^{2}, a_{R}^{i} \in \mathbb{R}, \forall R \in \mathscr{E}_{h}\right. \\
& \quad \text { if } R_{1} \text { and } R_{2} \text { share an edge } e, \text { then }\left.\int_{e} \xi\right|_{\partial R_{1}} d s=\left.\int_{e} \xi\right|_{\partial R_{2}} d s \\
& \left.\quad \text { and }\left.\int_{\partial R \cap \partial \Omega} \xi\right|_{\partial \Omega} d s=0\right\} .
\end{aligned}
$$

It will prove advantageous to understand some structure and properties of $M_{h}$.

Let the $P_{2}$-bubble function in $R \in \mathscr{E}_{h}$ be defined by

$$
\beta_{R}(x, y)=4-12\left(\frac{\left(x-x_{R}\right)^{2}}{h_{R x}^{2}}+\frac{\left(y-y_{R}\right)^{2}}{h_{R y}^{2}}\right),
$$

where $\left(x_{R}, y_{R}\right), h_{R x}$, and $h_{R y}$ are the center, $x$-length, and $y$-length of $R$, respectively. This bubble function vanishes at the two quadratic Gauss points on each edge (recall that the Gauss points on $[-1,1]$ are at $\pm 1 / \sqrt{3}$ ). Define the nonconforming spaces

$$
\begin{aligned}
& N_{h}=\left\{\xi:\left.\xi\right|_{R}=a_{R}^{1}+a_{R}^{2} x+a_{R}^{3} y+a_{R}^{4}\left(x^{2}-y^{2}\right), a_{R}^{i} \in \mathbb{R}, \forall R \in \mathscr{E}_{h} ;\right. \\
& \quad \text { if } R_{1} \text { and } R_{2} \text { share an edge } e, \text { then }\left.\int_{e} \xi\right|_{\partial R_{1}} d s=\left.\int_{e} \xi\right|_{\partial R_{2}} d s ; \\
& \left.\quad \text { and }\left.\int_{\partial R \cap \partial \Omega} \xi\right|_{\partial \Omega} d s=0\right\}, \\
& B_{h}=\left\{\xi:\left.\xi\right|_{R}=a_{R}^{5} \beta_{R}(x, y), a_{R}^{5} \in \mathbb{R}, \forall R \in \mathscr{E}_{h}\right\} .
\end{aligned}
$$

Namely, $N_{h}$ is a standard nonconforming space and $B_{h}$ is the set of $P_{2}$-bubble functions over $\mathscr{E}_{h}$.

Two-point Gaussian quadrature is exact on cubic functions. Therefore, we can rephrase the integral continuity constraint in $N_{h}$ (or in $M_{h}$ ) to say that on interior edges, the sum of the jump discontinuities in $\xi$ at each of the two quadratic Gauss points is zero, and on external boundary edges, the sum of $\xi$ at the two quadratic Gauss points is zero.

Lemma 1. The following three relations hold:

(i) For any $R \in \mathscr{E}_{h}, \nabla M_{h}(R)=V_{h}(R)$;

(ii) $M_{h}=N_{h} \oplus B_{h}$;

(iii) For any $R \in \mathscr{E}_{h},(\nabla \xi, \nabla \zeta)_{R}=0, \forall \xi \in N_{h}(R), \zeta \in B_{h}(R)$.

Note that (iii) holds if " $\nabla \xi$ " is replaced by any constant vector, since these are contained in $\nabla N_{h}(R)$.

Proof. Relations (i) and (ii) are trivial. Relation (iii) is a type of orthogonality. It can be seen after integration by parts,

$$
(\nabla \xi, \nabla \zeta)_{R}=-(\Delta \xi, \zeta)_{R}+(\nabla \xi \cdot \nu, \zeta)_{i j R}=0,
$$

since $\Delta \xi=0$ and $\nabla \xi \cdot \nu$ is constant. 
If $a$ is not diagonal, then we cannot easily exploit (i) and (iii). That is why we have assumed that $a$ is diagonal. In fact, we also need that the coefficients be piecewise constant. Fortunately, we can use a minor modification of the usual mixed method (2.1) consisting of projection of the coefficients into the space $W_{h}$. In that case, (i) and (iii) will prove to give us considerable computational savings, without any loss of accuracy (see $\S 7$ or $[10,14]$ ).

We need to maintain coercivity, so explicitly assume a somewhat stronger version of (1.2): for any $v \in\left(L^{2}(\Omega)\right)^{n}$ and $w \in L^{2}(\Omega)$,

$$
\left(\alpha_{h} v, v\right)+\left(b_{h} w, v\right)+\left(d_{h} w, w\right) \geq \kappa\left\{\|v\|_{\left(L^{2}(\Omega)\right)^{n}}^{2}+\left(d_{h} w, w\right)\right\}
$$

for some constant $\kappa>0$ independent of $h$, where $\alpha_{h}=\mathscr{P}_{W_{h}} a^{-1}, b_{h}=\mathscr{P}_{W_{h}} b$, and $d_{h}=\mathscr{P}_{W_{h}} d$. (This follows from (1.2) if $a$ and $d$ are sufficiently large compared to $b$, the coefficients are sufficiently smooth, and $h$ is sufficiently small.)

The mixed method for $(1.3)$ is then to find $\left(\sigma_{h}, u_{h}\right) \in V_{h} \times W_{h}$ such that

$$
\begin{aligned}
& \left(\nabla \cdot \sigma_{h}, w\right)+\left(d_{h} u_{h}, w\right)=\left(f_{h}, w\right), \quad \forall w \in W_{h}, \\
& \left(\alpha_{h} \sigma_{h}, v\right)-\left(u_{h}, \nabla \cdot v\right)+\left(b_{h} u_{h}, v\right)=\left(c_{h}, v\right), \quad \forall v \in V_{h},
\end{aligned}
$$

where $c_{h}=\mathscr{P}_{W_{h}} c$ and $f_{h}=\mathscr{P}_{W_{h}} f$. It is well known that $u_{h}$ approximates $u$ only to order one; therefore, various postprocessing techniques have been defined to improve the approximation. Let us define the following scheme (cf. Stenberg [22]): Find $\tilde{u}_{h} \in M_{h}$ such that in each $R \in \mathscr{E}_{h}$,

$$
\begin{aligned}
& \left(\tilde{u}_{h}-u_{h}, 1\right)_{R}=0, \\
& \left(\left(\nabla \tilde{u}_{h}+b_{h} u_{h}-c_{h}\right)+\alpha_{h} \sigma_{h}, \nabla \xi\right)_{R}=0, \quad \forall \xi \in M_{h}(R) .
\end{aligned}
$$

The equivalent nonconforming projection finite element method for approximating (1.1) has its coefficients modified accordingly. We find $\psi_{h} \in M_{h}$ such that

$$
\begin{aligned}
& \sum_{R \in \mathscr{E}_{h}}\left(\alpha_{h}^{-1}\left(\nabla \psi_{h}+b_{h} \mathscr{P}_{W_{h}} \psi_{h}-c_{h}\right), \nabla \xi\right)_{R}+\left(d_{h} \mathscr{P}_{W_{h}} \psi_{h}, \xi\right) \\
& \quad=\left(f_{h}, \xi\right), \quad \forall \xi \in M_{h} .
\end{aligned}
$$

Theorem 5. The solutions of (6.2)-(6.3) and (6.4) have the relationship

$$
\begin{aligned}
& \sigma_{h}=-\alpha_{h}^{-1}\left(\nabla \psi_{h}+b_{h} \mathscr{P}_{w_{h}} \psi_{h}-c_{h}\right), \\
& u_{h}=\mathscr{P}_{w_{h}} \psi_{h}, \\
& \tilde{u}_{h}=\psi_{h} .
\end{aligned}
$$

Proof. Since $\nabla M_{h}(R)=V_{h}(R), \tilde{\mathscr{P}}_{V_{h}}$ is unnecessary in (6.4) and (6.5), and so (6.5) and (6.6) follow from the general theory (for each fixed $\mathscr{E}_{h}$, we have fixed coefficients). Since $\psi_{h}$ satisfies (6.3), uniqueness of $\tilde{u}_{h}$ implies (6.7).

We give now a simple formula for computing the numerical flux $\sigma_{h}$ from an only slightly modified nonconforming method, (6.8) below.

Theorem 6. For each $R \in \mathscr{E}_{h}$, let

$$
\begin{aligned}
\gamma_{R} & =\left(d_{h} \mathscr{P}_{w_{h}} \beta_{R}-\alpha_{h}^{-1} \Delta \beta_{R}\right)^{-1}, \\
\omega_{R} & =1-d_{h} \gamma_{R} \mathscr{P}_{w_{h}} \beta_{R},
\end{aligned}
$$


and on $R$, define

$$
\begin{array}{ll}
\tilde{b}_{h}=\omega_{R} b_{h}, & \tilde{c}_{h}=c_{h}-b_{h} \gamma_{R} f_{h} \mathscr{P}_{W_{h}} \beta_{R}, \\
\tilde{d}_{h}=\omega_{R} d_{h}, & \tilde{f}_{h}=\omega_{R} f_{h} .
\end{array}
$$

Let $z_{h} \in N_{h}$ be the solution of

(6.8)

$\sum_{R \in \mathscr{E}_{h}}\left(\alpha_{h}^{-1}\left(\nabla z_{h}+\tilde{b}_{h} \mathscr{P}_{W_{h}} z_{h}-\tilde{c}_{h}\right), \nabla \xi\right)_{R}+\left(\tilde{d}_{h} \mathscr{P}_{W_{h}} z_{h}, \xi\right)=\left(\tilde{f}_{h}, \xi\right), \quad \forall \xi \in N_{h}$,

and $\zeta_{h} \in B_{h}$ be given by

$$
\left.\zeta_{h}(x, y)\right|_{R}=\left.\gamma_{R}\left(f_{h}-d_{h} \mathscr{P}_{W_{h}} z_{h}\right)\right|_{R} \beta_{R}(x, y) .
$$

Then $\psi_{h} \in M_{h}$ is the solution of (6.4) if, and only if, $\psi_{h}=z_{h}+\zeta_{h}$. Moreover, $\sigma_{h}$ at a point $(x, y) \in R \in \mathscr{E}_{h}$ is evaluated by the formula

$$
\begin{aligned}
\sigma_{h}(x, y)=-\alpha_{h}^{-1}\left\{\nabla z_{h}(x, y)+\tilde{b}_{h}\right. & \left.\mathscr{P}_{W_{h}} z_{h}\right|_{R}-\tilde{c}_{h} \\
& \left.+\left.\gamma_{R}\left(f_{h}-d_{h} \mathscr{P}_{W_{h}} z_{h}\right)\right|_{R} \nabla \beta_{R}(x, y)\right\} .
\end{aligned}
$$

Proof. We begin by noting that $0<\omega_{R}<1$, and $\omega_{R} \rightarrow 1$ as $h \rightarrow 0$. In fact, since $a, d$, and $\Omega$ are bounded, $\omega_{R} \geq \omega_{*}>0$ for some constant $\omega_{*}$ independent of $h$. As a consequence, (6.1) holds with $\tilde{b}_{h}$ and $\tilde{d}_{h}$ replacing $b_{h}$ and $d_{h}$, respectively. Therefore, (6.8) is well posed.

We exploit the orthogonality (iii) of Lemma 1 to obtain the theorem. Let $\psi_{h}$ be the solution to (6.4) and let $\psi_{h}=z_{h}+\zeta_{h}$ for some $z_{h} \in N_{h}$ and $\zeta_{h} \in B_{h}$. We must show that (6.8)-(6.9) hold.

Restrict to a test function $\xi \in B_{h}$ in (6.4), and use orthogonality to see that (6.11)

$\left(\alpha_{h}^{-1} \nabla \zeta_{h}, \nabla \xi\right)_{R}+\left(d_{h} \mathscr{P}_{W_{h}} \zeta_{h}, \xi\right)_{R}=\left(f_{h}-d_{h} \mathscr{P}_{W_{h}} z_{h}, \xi\right)_{R},\left.\quad \forall \xi \in B_{h}\right|_{R}, \quad R \in \mathscr{E}_{h}$.

Integrate by parts the first term on the left-hand side to obtain that

$$
d_{h} \mathscr{P}_{W_{h}} \zeta_{h}-\alpha_{h}^{-1} \Delta \zeta_{h}=\left.\left(f_{h}-d_{h} \mathscr{P}_{W_{h}} z_{h}\right)\right|_{R} \quad \text { in each } R \in \mathscr{E}_{h},
$$

since the boundary term is zero by appeal to Gaussian quadrature. It follows from the definition of $B_{h}$ that $\zeta_{h}$ is given by (6.9).

In (6.4), restrict now to $\xi \in N_{h}$ and use (6.9) and orthogonality to obtain (6.8) for $z_{h}$, since in each $R \in \mathscr{E}_{h}$,

$$
\begin{aligned}
\mathscr{P}_{W_{h}} \psi_{h} & =\mathscr{P}_{W_{h}} z_{h}+\mathscr{P}_{W_{h}} \zeta_{h}=\mathscr{P}_{W_{h}} z_{h}+\gamma_{R}\left(f_{h}-d_{h} \mathscr{P}_{W_{h}} z_{h}\right) \mathscr{P}_{W_{h}} \beta_{R} \\
& =\omega_{R} \mathscr{P}_{W_{h}} z_{h}+\gamma_{R} f_{h} \mathscr{P}_{W_{h}} \beta_{R} .
\end{aligned}
$$

Conversely, we obtain (6.4) from (6.8)-(6.9) and unisolvence.

Finally, from (6.5) and (6.12),

$$
\sigma_{h}=-\alpha_{h}^{-1}\left\{\nabla z_{h}+b_{h}\left[\omega_{R} \mathscr{P}_{W_{h}} z_{h}+\gamma_{R} f_{h} \mathscr{P}_{W_{h}} \beta_{R}\right]-c_{h}+\nabla \zeta_{h}\right\}
$$

and so (6.10) follows.

We end this section with three remarks. First, if $u_{h}$ is needed, it is given by (6.12) (recall (6.6)). However, since $\psi_{h}$ approximates $u$ to a higher order of accuracy than $u_{h}$, as shown in the next theorem, the use of (6.12) seems inadvisable. Secondly, if the Lagrange multipliers for the mixed method are 
desired, they are the average value of $\psi_{h}$ or $z_{h}$ on each edge. Thirdly, if $R$ is a square and $b=c=d=0,(6.10)$ is simply

$$
\sigma_{h}=-\alpha_{h}^{-1} \nabla z_{h}+\left.\frac{1}{2} \mathscr{P}_{W_{h}} f\right|_{R}\left(x-x_{R}, y-y_{R}\right), \quad \forall(x, y) \in R \in \mathscr{E}_{h},
$$

which is the same form as in the case of triangular mixed finite elements [18, $11]$.

\section{ERROR ESTIMATES}

Denote by $\|\cdot\|_{j, S}$ the norm of $H^{j}(S)$, where we omit $j$ if $j=0$ and $S$ if $S=\Omega$. We have the following theorem.

Theorem 7. If $u$ and $\sigma$ solve (1.1), $u_{h}$ and $\sigma_{h}$ solve (6.2), and $\psi_{h}$ solves (6.4), then there is a constant $C$ independent of $h$ such that

$$
\begin{aligned}
& \text { (7.1) }\left\|\sigma-\sigma_{h}\right\|+\left\|u-u_{h}\right\| \leq C\left(\|f\|,\|a\|_{W^{1, \infty}(\Omega)},\|b\|_{1},\|c\|_{1}\right) h, \\
& \text { (7.2) }\left\|\nabla \cdot\left(\sigma-\sigma_{h}\right)\right\| \leq C\left(\|f\|_{1},\|a\|_{W^{1, \infty}(\Omega)},\|b\|_{1},\|c\|_{1}\right) h, \\
& \text { (7.3) }\left\|\mathscr{P}_{W_{h}} u-u_{h}\right\| \leq C\left(\|f\|_{1},\|a\|_{W^{1, \infty}(\Omega)},\|b\|_{W^{1, \infty}(\Omega)},\|c\|_{1},\|d\|_{W^{1, \infty}(\Omega)}\right) h^{2}, \\
& \text { (7.4) }\left(\sum_{R \in \mathscr{E}_{h}}\left\|\nabla u-\nabla \psi_{h}\right\|_{R}^{2}\right)^{1 / 2} \leq C\left(\|f\|,\|a\|_{W^{1, \infty}(\Omega)},\|b\|_{1},\|c\|_{1}\right) h, \\
& \text { (7.5) }\left\|u-\psi_{h}\right\| \leq C\left(\|f\|_{1},\|a\|_{W^{1, \infty}(\Omega)},\|b\|_{W^{1, \infty}(\Omega)},\|c\|_{1},\|d\|_{W^{1, \infty}(\Omega)}\right) h^{2} .
\end{aligned}
$$

Proof. Results (7.1)-(7.3) are essentially known [14]. They can be obtained by a careful application of the techniques of Douglas and Roberts [15]. To handle the modified coefficients, we must recognize that for $s=0,1$ and $1 \leq j \leq \infty$,

$$
\left\|P_{W_{h}} \phi-\phi\right\|_{W^{-s, j(\Omega)}} \leq C\|\phi\|_{W^{1, j}(\Omega)} h^{1+s} .
$$

We also use elliptic regularity to obtain that

$$
\|u\|_{2} \leq C\|f\|_{0} \text { and }\|\nabla \cdot u\|_{1} \leq C\|f\|_{1},
$$

and a duality argument to obtain (7.3).

Results (7.4)-(7.5) follow from the use of an abstract theorem concerning error estimates between $u$ and $\psi_{h}$ (see [11, Theorem 2.2]). However, a simpler approach is to note by equivalence from (6.3) that

(7.7) $\left(u-\psi_{h}, w\right)=\left(P_{W_{h}} u-u_{h}, w\right), \quad \forall w \in W_{h}$,

$$
\begin{aligned}
& \left(\nabla\left(u-\psi_{h}\right)+b_{h}\left(u-u_{h}\right)+\alpha_{h}\left(\sigma-\sigma_{h}\right), \nabla \xi\right)_{R} \\
& \quad=\left(\left(b_{h}-b\right) u+c-c_{h}+\left(\alpha_{h}-a^{-1}\right) \sigma, \nabla \xi\right)_{R}, \quad \forall \xi \in M_{h}(R) \text { and } R \in \mathscr{E}_{h} .
\end{aligned}
$$

Estimate in a straightforward way the second elliptic equation to obtain (7.4) from (7.1) and (7.6). Use (7.7) to obtain that

$$
\left\|u-\psi_{h}\right\|_{0, R} \leq C\left\{\left\|\nabla\left(u-\psi_{h}\right)\right\|_{0, R} h+\left\|\mathscr{P}_{w_{h}} u-u_{h}\right\|_{0, R}\right\}
$$

and then apply (7.3) and (7.4) to finish the proof.

\section{A MULTIGRID SOLUTION ALGORITHM}

In this section we develop a multigrid algorithm for the nonconforming method (6.4) and the mixed method (6.2). We need to assume a structure 
to our family of partitions. Let $h_{0}$ and $\mathscr{E}_{h_{0}}=\mathscr{E}_{0}$ be given. For each integer $k \geq 1$, let $h_{k}=2^{-k} h_{0}$ and $\mathscr{E}_{h_{k}}=\mathscr{E}_{k}$ be constructed by connecting the midpoints of the edges of the rectangles in $\mathscr{E}_{k-1}$. In this section (and the appendix) only, we will replace subscript $h_{k}$ simply by subscript $k$. Since the intergrid transfer operators below do not preserve either the energy or the $L^{2}$-norm, as noted in [3], the standard argument of convergence for $V$-cycles does not carry over directly. So, only a $W$-cycle, full multigrid algorithm will be defined here. Since mixed methods are designed to approximate well the flux variable $\sigma$, and since it is of primary interest in many applications, we develop the multigrid algorithm with emphasis on the calculation of this variable. We assume in this section that $b=c=0$.

With this in mind, we now take advantage of the factorization of the system (6.4) into (6.8) and (6.11). For each $k$, let

$$
\begin{aligned}
& \tilde{a}_{k}(\xi, \zeta)=\sum_{R \in \mathscr{E}_{k}}\left(\alpha_{k}^{-1} \nabla \xi, \nabla \zeta\right)_{R}+\left(\tilde{d}_{k} \mathscr{P}_{W_{k}} \xi, \zeta\right), \\
& a_{k}(\xi, \zeta)=\sum_{R \in \mathscr{E}_{k}}\left(\alpha_{k}^{-1} \nabla \xi, \nabla \zeta\right)_{R}+\left(d_{k} \mathscr{P}_{W_{k}} \xi, \zeta\right), \quad \forall \xi, \zeta \in M_{k} .
\end{aligned}
$$

Then (6.8) asks for $z_{k} \in N_{k}$ such that

$$
\tilde{a}_{k}\left(z_{k}, \xi\right)=\left(\tilde{f}_{k}, \xi\right), \quad \forall \xi \in N_{k},
$$

and (6.11) asks for $\zeta_{k} \in B_{k}$ such that

$$
a_{k}\left(\zeta_{k}, \varphi\right)=\left(f_{k}-d_{k} \mathscr{P}_{W_{k}} z_{k}, \varphi\right), \quad \forall \varphi \in B_{k} .
$$

For $k=1,2, \ldots$, solutions to problem (8.2) can be obtained directly, since $B_{k}$ has no continuity constraints across element boundaries; therefore, we define a multigrid procedure for (8.1) only.

Standard inverse estimates yield that the spectral radius of the operator $\tilde{a}_{k}$ on $N_{k} \times N_{k}$ is bounded above: there exists a constant $C_{1}$ independent of $k$ such that

$$
\text { spectral radius of } \tilde{a}_{k} \text { on } N_{k} \times N_{k} \leq C_{1} h_{k}^{-2} \text {. }
$$

Note that, since $N_{k-1} \not \subset N_{k}$, these spaces are not nested. It is well known that natural injection operators do not work for nonnested finite element spaces. Thus, we need to introduce special intergrid transfer operators. Following [4], we define the coarse-to-fine intergrid transfer operators $I_{k-1}^{k}: N_{k-1} \rightarrow N_{k}$ as follows. If $\xi \in N_{k-1}$ and $e$ is an edge of a rectangle in $\mathscr{E}_{k}$, then $I_{k-1}^{k} \xi \in N_{k}$ is defined by

$$
\frac{1}{|e|} \int_{e} I_{k-1}^{k} \xi d \sigma= \begin{cases}0 & \text { if } e \subset \partial \Omega, \\ \frac{1}{|e|} \int_{e} \xi d \sigma & \text { if } e \not \subset \partial R \text { for any } R \in \mathscr{E}_{k-1}, \\ \frac{1}{2|e|}\left\{\int_{e}\left(\left.\xi\right|_{R_{1}}+\left.\xi\right|_{R_{2}}\right) d \sigma\right\} & \text { if } e \subset \partial R_{1} \cap \partial R_{2} \text { for some } \\ & R_{1}, R_{2} \in \mathscr{E}_{k-1} .\end{cases}
$$

The multigrid algorithm for obtaining approximate solutions $\widehat{z}_{k} \in N_{k}$ to problem (8.1) is given in terms of the $k$ th-level multigrid step, defined below, which yields the result $\mathrm{MG}\left(k, z^{k}, \tilde{f}_{k}\right) \in N_{k}$ as an approximate solution to (8.1) 
from the initial guess $z^{k} \in N_{k}$. Let $r$ be a positive integer independent of $k$, which denotes the number of multigrid iterations in (ii) below. The overall multigrid algorithm is defined sequentially for each $k$ as follows:

(8.5a) For $k=1, \widehat{z}_{1}=\mathrm{MG}\left(1, \cdot, \tilde{f}_{1}\right)$ is obtained by a direct method;

(8.5b) For $k \geq 2, \widehat{z}_{k}$ is obtained recursively by

(i) $z_{0}^{k}=I_{k-1}^{k} \widehat{z}_{k-1}$,

(ii) $z_{\ell}^{k}=\mathrm{MG}\left(k, z_{\ell-1}^{k}, \tilde{f}_{k}\right), 1 \leq \ell \leq r$,

(iii) $\widehat{z}_{k}=z_{r}^{k}$.

The multigrid step is defined for $k=1$ and $F \in N_{1}^{\prime}$ as $\operatorname{MG}(1, \cdot, F)=z_{1}$, where $z_{1}$ is obtained directly as the solution to

$$
\tilde{a}_{1}\left(z_{1}, \xi\right)=(F, \xi), \quad \forall \xi \in N_{1} .
$$

For $k \geq 2, z \in N_{k}$, and $F \in N_{k}^{\prime}$,

$$
\text { (MG) } \operatorname{MG}(k, z, F)=S(k, z, F)+C(k, S(k, z, F), F)
$$

is calculated by means of the smoothing step

(S) $S\left(k, g_{0}, F\right)=g_{m}$, where $m$ is the number of smoothing steps and the approximation $g_{j} \in N_{k}, j=1,2, \ldots, m$, is defined recursively from the initial guess $g_{0}$ by the equations

$$
\left(g_{j}-g_{j-1}, \xi\right)=C_{1}^{-1} h_{k}^{2}\left((F, \xi)-\tilde{a}_{k}\left(g_{j-1}, \xi\right)\right), \quad \forall \xi \in N_{k}, j=1, \ldots, m,
$$

and the correction step

(C) $C\left(k, g_{m}, F\right)=I_{k-1}^{k} q_{p}$, where $q_{j} \in N_{k-1}, j=0, \ldots, p(p=2$ or 3 ), is defined recursively from $q_{0}=0$ by

$$
\begin{aligned}
& q_{j}=\operatorname{MG}\left(k-1, q_{j-1}, F\right), \quad j=1, \ldots, p, \\
& (F, \xi)=\left(F, I_{k-1}^{k} \xi\right)-\tilde{a}_{k}\left(g_{m}, I_{k-1}^{k} \xi\right), \quad \forall \xi \in N_{k-1} .
\end{aligned}
$$

From (6.10), the multigrid approximate solution $\widehat{\sigma}_{k}$ to $\sigma_{k}$ is defined in $R \in \mathscr{E}_{k}$ by

$$
\widehat{\sigma}_{k}=-\alpha_{k}^{-1}\left\{\nabla \widehat{z}_{k}+\left.\gamma_{R}\left(f_{k}-d_{k} \mathscr{P}_{W_{k}} \widehat{z}_{k}\right)\right|_{R} \nabla \beta_{R}(x, y)\right\} .
$$

The standard argument $[2,3,4]$ for the convergence analysis of the multigrid algorithm (8.5) applies here if we prove that $I_{k-1}^{k}$ is bounded and reduces to the natural injection on continuous bilinear functions. Although the second fact is false, it is true after a modification of the definition of $I_{k-1}^{k}$ given in the appendix (the modified definition is equivalent to the original on $N_{k-1}$ ). The first fact together with the following lemma will be shown in the appendix.

Lemma 2. If $m$ and $r$ in the multigrid algorithm are sufficiently large, there is a constant $C\left(\|a\|_{W^{1, \infty}(\Omega)},\|d\|_{W^{1, \infty}(\Omega)}\right)$ independent of $k$ such that

$$
\begin{aligned}
& \left\|z_{k}-\widehat{z}_{k}\right\|+\left(\sum_{R \in \mathscr{E}_{k}}\left\|\nabla\left(z_{k}-\widehat{z}_{k}\right)\right\|_{R}^{2}\right)^{1 / 2} \leq C h_{k}\|f\|, \\
& \left\|z_{k}-\widehat{z}_{k}\right\| \leq C h_{k}^{2}\|f\|_{1} .
\end{aligned}
$$


Theorem 8. If $m$ and $r$ in the multigrid algorithm are sufficiently large, then there is a constant $C\left(\|a\|_{W^{1, \infty}(\Omega)},\|d\|_{W^{1, \infty}(\Omega)}\right)$ such that

$$
\begin{aligned}
& \left\|\sigma_{k}-\widehat{\sigma}_{k}\right\| \leq C h_{k}\|f\|, \\
& \left\|\sigma-\widehat{\sigma}_{k}\right\| \leq C h_{k}\|f\| .
\end{aligned}
$$

Proof. Equations (6.10), (8.6), and (8.7) imply equation (8.9), since $\gamma_{R}=\mathscr{O}\left(h_{k}^{2}\right)$ and $\left\|\nabla \beta_{R}\right\|=\mathscr{O}\left(h_{k}^{-1}\right)$. Equation (8.10) follows with (7.1) (the bound is proportional to $\|f\|$ because $c=0)$.

It can be seen that the total work performed in obtaining $\widehat{z}_{k}$ is $O\left(n_{k}\right)$ [2]; thus, the cost to compute $\widehat{\sigma}_{k}$ is also $O\left(n_{k}\right)$.

Since $\widehat{\sigma}_{k}$ belongs to

$$
\widehat{V}_{k}=\left\{v:\left.v\right|_{R}=\left(a_{R}^{1}+a_{R}^{2} x, a_{R}^{3}+a_{R}^{4} y\right), a_{R}^{i} \in \mathbb{R}, \forall R \in \mathscr{E}_{k}\right\},
$$

but not necessarily to $V_{k}$, following [4], we introduce the averaging operator $\Lambda_{k}: \widehat{V}_{k} \rightarrow V_{k}$. Let $e$ be an edge of $R \in \mathscr{E}_{k}$ and $n_{e}$ be a unit outer normal to $e$. For $v \in \widehat{V}_{k}$, if $e \subset \partial \Omega$, then $\left.\left(\Lambda_{k} v \cdot \nu\right)\right|_{e}=\left.\left(\left.v\right|_{R} \cdot \nu\right)\right|_{e}$; if $e$ is the common edge of $R_{1}$ and $R_{2} \in \mathscr{E}_{k}$, then

$$
\left.\left(\Lambda_{k} v \cdot \nu_{R_{1}}\right)\right|_{e}=\frac{1}{2}\left(\left.\left(\left.v\right|_{R_{1}} \cdot \nu_{R_{1}}\right)\right|_{e}+\left.\left(\left.v\right|_{R_{2}} \cdot \nu_{R_{1}}\right)\right|_{e}\right) \text {. }
$$

Thus, $\Lambda_{k}$ restricted to $V_{k}$ is the identity. The next result follows from this definition and Theorem 8.

Theorem 9. There is a constant $C$ such that

$$
\left\|\Lambda_{k} v\right\| \leq C\|v\|, \quad \forall v \in \widehat{V}_{k} .
$$

Moreover, under the conditions of Theorem 8, there is a constant

$$
C\left(\|a\|_{W^{1, \infty}(\Omega)},\|d\|_{W^{1, \infty}(\Omega)}\right)
$$

such that

$$
\begin{aligned}
& \left\|\sigma_{k}-\Lambda_{k} \widehat{\sigma}_{k}\right\| \leq C h_{k}\|f\|, \\
& \left\|\sigma-\Lambda_{k} \widehat{\sigma}_{k}\right\| \leq C h_{k}\|f\| .
\end{aligned}
$$

The final result in this section concerns $\psi_{k}$, defined by (6.4). Recall that $\zeta_{k}$ is the solution to (8.2), and define

$$
\widehat{\psi}_{k}=\widehat{z}_{k}+\zeta_{k} \text {. }
$$

Since $\psi_{k}-\widehat{\psi}_{k}=z_{k}-\widehat{z}_{k}$, we have the following from Lemma 2 and (7.5).

Theorem 10. If the assumptions of Theorem 8 are satisfied, then there is a constant $C\left(\|a\|_{W^{1, \infty}(\Omega)},\|d\|_{W^{1, x}(\Omega)}\right)$ such that

$$
\left\|\psi_{k}-\widehat{\psi}_{k}\right\|+\left(\sum_{R \in \mathscr{E}_{k}}\left\|\nabla\left(\psi_{k}-\widehat{\psi}_{k}\right)\right\|_{R}^{2}\right)^{1 / 2} \leq C h_{k}\|f\| .
$$

Moreover, if $f \in H^{1}(\Omega)$,

$$
\begin{aligned}
& \left\|\psi_{k}-\widehat{\psi}_{k}\right\| \leq C h_{k}^{2}\|f\|_{1}, \\
& \left\|u-\widehat{\psi}_{k}\right\| \leq C h_{k}^{2}\|f\|_{1} .
\end{aligned}
$$


An averaging process similar to that for $\widehat{\sigma}_{k}$ can be defined for $\widehat{\psi}_{k}$. The multigrid algorithms developed in this section for the rectangular elements can be extended to the lowest-order triangular elements and the results in Theorems $8-10$ remain valid.

\section{EXTENSION TO RECTANGULAR PARALLELEPIPEDS}

Let now $\Omega$ be a polygonal domain in $\mathbb{R}^{3}$ and $\mathscr{E}_{h}$ be a decomposition of $\Omega$ into rectangular parallelepipeds having maximum diameter $h$ and oriented along the coordinate axes. Again assume that $a$ is a scalar, $\Gamma_{2}=\varnothing$, and $g=0$.

We consider the lowest-order Raviart-Thomas-Nedelec space [19] $W_{h} \times V_{h}$ defined over $\mathscr{E}_{h}$ (equivalently, the lowest-order Brezzi-Douglas-Fortin-Marini space [7]).

Let $M_{h}$ be the nonconforming space introduced in $\S \S 5.4$ and 5.6 above. We obtain Lemma 1 in $\S 6$ provided that we redefine

$$
\begin{gathered}
N_{h}=\left\{\xi:\left.\xi\right|_{R}=a_{R}^{1}+a_{R}^{2} x+a_{R}^{3} y+a_{R}^{4} z+a_{R}^{5}\left(x^{2}-y^{2}\right)+a_{R}^{6}\left(x^{2}-z^{2}\right),\right. \\
a_{R}^{i} \in \mathbb{R}, \forall R \in \mathscr{E}_{h} ; \text { if } R_{1} \text { and } R_{2} \text { share a face } e, \\
\text { then } \left.\left.\int_{e} \xi\right|_{\partial R_{1}} d s=\left.\int_{e} \xi\right|_{\partial R_{2}} d s ; \text { and }\left.\int_{\partial R \cap \partial \Omega} \xi\right|_{\partial \Omega} d s=0\right\}, \\
B_{h}=\left\{\xi:\left.\xi\right|_{R}=a_{R}^{7} \beta_{R}(x, y), a_{R}^{7} \in \mathbb{R}, \forall R \in \mathscr{E}_{h}\right\}
\end{gathered}
$$

where now the $P_{2}$-bubble function in each $R \in \mathscr{E}_{h}$ is

$$
\beta_{R}(x, y, z)=5-12\left(\frac{\left(x-x_{R}\right)^{2}}{h_{R x}^{2}}+\frac{\left(y-y_{R}\right)^{2}}{h_{R y}^{2}}+\frac{\left(z-z_{R}\right)^{2}}{h_{R z}^{2}}\right),
$$

which is equal to zero at the four tensor product quadratic Gauss points on each face.

With these modifications, we again have the equivalence between the solutions of (6.2)-(6.3) and (6.4) in the sense of Theorem 5. Theorems 6 and 7 hold as well; moreover, if $\mathscr{E}_{h_{1}}$ is given and each $\mathscr{E}_{h_{k+1}}$ is a regular refinement of $\mathscr{E}_{h_{k}}$ into eight times as many elements, then the results in $\S 8$ remain valid.

\section{EXTENSION TO SIMPLICES}

Let now $\mathscr{E}_{h}$ be a partition of $\Omega$ into simplices, and again assume that $a$ is a scalar, $\Gamma_{2}=\varnothing$, and $g=0$. The lowest-order Raviart-Thomas-Nedelec space $V_{h}[21,19]$ defined over $\mathscr{E}_{h}$ is given by

$$
\begin{aligned}
V_{h}= & \left\{v:\left.v\right|_{E}=\left(a_{E}^{1}+a_{E}^{2} x, a_{E}^{3}+a_{E}^{2} y, a_{E}^{4}+a_{E}^{2} z\right), a_{E}^{i} \in \mathbb{R}, \forall E \in \mathscr{E}_{h} ;\right. \\
\left.v \cdot n \text { is continuous at the interelement faces of } \mathscr{E}_{h}\right\}, & \\
W_{h}= & \left\{w:\left.w\right|_{E} \text { is constant, } \forall E \in \mathscr{E}_{h}\right\}, \\
L_{h}= & \left\{\mu:\left.\mu\right|_{e} \text { is constant, } \forall e \in \partial \mathscr{E}_{h}\right\} .
\end{aligned}
$$

We define the nonconforming space $N_{h}$ by

$$
\begin{aligned}
N_{h}= & \left\{\xi:\left.\xi\right|_{E}=a_{E}^{1}+a_{E}^{2} x+a_{E}^{3} y+a_{E}^{4} z, a_{E}^{i} \in \mathbb{R}, \forall E \in \mathscr{E}_{h} ; \text { if } E_{1} \text { and } E_{2}\right. \\
& \text { share a face } \left.e, \text { then }\left.\int_{e} \xi\right|_{\partial E_{1}} d s=\left.\int_{e} \xi\right|_{\partial E_{2}} d s ; \text { and }\left.\int_{\partial E \cap \partial \Omega} \xi\right|_{\partial \Omega} d s=0\right\} .
\end{aligned}
$$


For each $E \in \mathscr{E}_{h}$, let $\hat{\ell}_{i}, i=1,2,3,4$, denote the barycentric coordinates of a point in the simplex. These functions are the unique affine functions that take the value one at vertex $i$, and the value zero on the opposite face. The $P_{2}$-bubble function takes the form

$$
\beta_{E}(x, y, z)=1-2\left(\hat{\ell}_{1}^{2}+\hat{\ell}_{2}^{2}+\hat{\ell}_{3}^{2}+\hat{\ell}_{4}^{2}\right), \quad \forall(x, y, z) \in E .
$$

This quadratic bubble function has vanishing integral over each face. Let

$$
B_{h}=\left\{\xi:\left.\xi\right|_{E}=a_{E}^{5} \beta_{E}, a_{E}^{5} \in \mathbb{R}\right\}
$$

and $M_{h}=N_{h} \oplus B_{h}$. This $M_{h}$ satisfies the conditions of Theorem 4 (in particular, (DF) are unisolvent).

We have an analogue of Lemma 1.

Lemma 3. The following two relations hold:

(i) For any $E \in \mathscr{E}_{h}, \nabla N_{h}(E) \subset V_{h}(E)$;

(ii) For any $E \in \mathscr{E}_{h},(\nabla \xi, \nabla \zeta)_{E}=0, \forall \xi \in N_{h}(E), \zeta \in B_{h}(E)$.

Proof. For (i), $\nabla N_{h}(E)=\left(P_{0}(E)\right)^{3} \subset V_{h}(E)$. For (ii), integrate by parts and use that $\Delta \xi=0$.

To exploit this orthogonality, we will assume as in $\S 6$ that the coefficients are projected into the space $W_{h}$. So assume (6.1) and take (6.2). As an analogue of (6.3), we define $\tilde{u}_{h} \in M_{h}$ such that on each $E$,

$$
\begin{aligned}
& \left(\tilde{u}_{h}-u_{h}, 1\right)_{E}=0, \\
& \left(\left(\tilde{\mathscr{P}}_{V_{h}} \nabla \tilde{u}_{h}+b_{h} u_{h}-c_{h}\right)+\alpha_{h} \sigma_{h}, \nabla \xi\right)_{E}=0, \quad \forall \xi \in M_{h}(E) .
\end{aligned}
$$

(The existence of $\tilde{u}_{h}$ follows easily from Lemma 3 ).

Note that for any $\xi \in M_{h}$, we can write $\xi_{h}=z_{h}+\zeta_{h}$, where $z_{h} \in N_{h}$ and $\zeta_{h} \in B_{h}$. Then $\tilde{\mathscr{P}}_{V_{h}} \nabla \xi_{h}=\nabla z_{h}+\tilde{\mathscr{P}}_{V_{h}} \nabla \zeta_{h}$. The equivalent nonconforming projection finite element method for approximating (6.2) is to find $\psi_{h} \in M_{h}$ such that

$$
\begin{aligned}
& \sum_{E \in \mathscr{E}_{h}}\left(\alpha_{h}^{-1}\left(\tilde{\mathscr{P}}_{V_{h}} \nabla \psi_{h}+b_{h} \mathscr{P}_{W_{h}} \psi_{h}-c_{h}\right), \nabla \xi\right)_{E}+\left(d_{h} \mathscr{P}_{W_{h}} \psi_{h}, \xi\right) \\
& \quad=\left(f_{h}, \xi\right), \quad \forall \xi \in M_{h} .
\end{aligned}
$$

Then Theorem 5 holds, provided (6.5) is replaced by

$$
\sigma_{h}=-\alpha_{h}^{-1}\left(\tilde{\mathscr{P}}_{V_{h}} \nabla \psi_{h}+b_{h} \mathscr{P}_{W_{h}} \psi_{h}-c_{h}\right) .
$$

Theorem 6 also holds, provided that now

$$
\gamma_{R}=\left(d_{h} \mathscr{P}_{W_{h}} \beta_{R}-\alpha_{h}^{-1} \nabla \cdot \tilde{P}_{V_{h}} \nabla \beta_{R}\right)^{-1}
$$

and (6.10) is replaced by

$$
\begin{aligned}
\sigma_{h}(x, y)=-\alpha_{h}^{-1}\left\{\nabla z_{h}(x, y)\right. & +\left.\tilde{b}_{h} \mathscr{P}_{W_{h}} z_{h}\right|_{R}-\tilde{c}_{h} \\
& \left.+\left.\gamma_{R}\left(f_{h}-d_{h} \mathscr{P}_{W_{h}} z_{h}\right)\right|_{R} \tilde{\mathscr{P}}_{V_{h}} \nabla \beta_{R}(x, y)\right\} .
\end{aligned}
$$

The convergence result in $\S 7$ also holds. In the case of equilateral simplices, this can be seen as before since then $\nabla B_{h}(E) \subset V_{h}(E)$ and the projection operator $\tilde{\mathscr{P}}_{V_{h}}$ in (10.3) can be removed. In the general case the convergence 
result can be shown using the ideas given in [4] (that is, we show that $\left\|\tilde{\mathscr{P}}_{V_{h}} \nabla \xi\right\|$ and $\|\nabla \xi\|$ are equivalent norms for $\xi \in N_{h}$, and we use the interpolant of $u$ into $N_{h}$ as an intermediary). Finally, results analogous to those in $\S 8$ are valid.

\section{EXTENSION TO PRISMS}

Let now $\Omega$ be of the form $\Omega=G \times[0,1]$ with $G \subset \mathbb{R}^{2}$ and $\mathscr{E}_{h}$ be a partition of $\Omega$ into prisms with three vertical edges parallel to the $z$-axis and two horizontal faces in the $(x, y)$-plane. Let $E=T \times\left(z_{E a}, z_{E b}\right)$ denote such a prism, of height $h_{E z}=z_{E b}-z_{E a}$. Again, $\hat{\ell}_{i}, i=1,2,3$, denote the barycentric coordinates of a point in the triangle $T$. In this section, we again assume that $a$ is a scalar, $\Gamma_{2}=\varnothing$, and $g=0$.

The lowest-order prismatic space $V_{h}$ [20] defined over $\mathscr{E}_{h}$ is given by

$$
\begin{aligned}
V_{h}=\left\{v:\left.v\right|_{E}=\left(a_{E}^{1}+a_{E}^{2} x, a_{E}^{3}+a_{E}^{2} y, a_{E}^{4}+a_{E}^{5} z\right), a_{E}^{i} \in \mathbb{R}, \forall E \in \mathscr{E}_{h} ;\right. \\
\left.v \cdot n \text { is continuous at the interelement faces of } \mathscr{E}_{h}\right\}, \\
W_{h}=\left\{w:\left.w\right|_{E} \text { is constant, } \forall E \in \mathscr{E}_{h}\right\}, \\
L_{h}=\left\{\mu:\left.\mu\right|_{e} \text { is constant, } \forall e \in \partial \mathscr{E}_{h}\right\} .
\end{aligned}
$$

The nonconforming space $N_{h}$ is defined by

$$
\begin{aligned}
& N_{h}=\left\{\xi:\left.\xi\right|_{E}=a_{E}^{1}+a_{E}^{2} x+a_{E}^{3} y+a_{E}^{4} z+a_{E}^{5}\left(x^{2}+y^{2}-2 z^{2}\right), a_{E}^{i} \in \mathbb{R}, \forall E \in \mathscr{E}_{h} ;\right. \\
& \quad \text { if } E_{1} \text { and } E_{2} \text { share a face } e, \text { then }\left.\int_{e} \xi\right|_{\partial E_{1}} d s=\left.\int_{e} \xi\right|_{\partial E_{2}} d s ; \\
& \left.\quad \text { and }\left.\int_{\partial E \cap \partial \Omega} \xi\right|_{\partial \Omega} d s=0\right\}, \\
& B_{h}=\left\{\xi:\left.\xi\right|_{E}=a_{E}^{6} \beta_{E}, a_{E}^{6} \in \mathbb{R}\right\},
\end{aligned}
$$

where the $P_{2}$-bubble function takes the form

$$
\beta_{E}(x, y, z)=3-4\left(\hat{\ell}_{1}^{2}+\hat{\ell}_{2}^{2}+\hat{\ell}_{3}^{2}\right)-\frac{4}{h_{E z}^{2}}\left(z-\frac{z_{E a}+z_{E b}}{2}\right)^{2}, \quad \forall(x, y, z) \in E,
$$

so that its integral over each face vanishes. Finally, $M_{h}=N_{h} \oplus B_{h}$.

We have Lemma 3 and the results for simplices of the last section hold also for prisms.

\section{Appendix. Proof of Lemma 2}

We prove Lemma 2 of $\S 8$ in this appendix. Recall that here $b=c=0$. From $\S 6$, note that on the $k$ th mesh

$$
\beta_{R}=\mathscr{O}(1), \quad \nabla \beta_{R}=\mathscr{O}\left(h_{k}^{-1}\right), \quad \Delta \beta_{R}=\mathscr{O}\left(h_{k}^{-2}\right),
$$

so

$$
\left|\gamma_{R}\right| \leq C h_{k}^{2} \text { and }\left|\omega_{R}-1\right| \leq C h_{k}^{2}
$$

where $C$ depends only on the bounds for $a$ and $d$. (In general, without further comment, we will assume that the generic constant $C$ may depend on $\|a\|_{W^{1, \infty}(\Omega)}$ and $\|d\|_{W^{1, \infty}(\Omega)}$ in this appendix.) Since $\left\|\tilde{f}_{k}-f_{k}\right\| \leq C h_{k}^{2}\|f\|$, we can replace $\tilde{f}_{k}$ by $f_{k}$ up to the second order in $h_{k}$. A similar statement holds for $\tilde{d}_{k}$ and $d_{k}$. 
For each $k$, define the energy norm

$$
\|\xi\|_{k}=\left(\tilde{a}_{k}(\xi, \xi)\right)^{1 / 2} .
$$

(This is equivalent to the $H^{1}(\Omega)$-norm by (6.1) and a Poincare inequality.) Standard arguments for the error in approximating (1.1) by a nonconforming method are easily combined with arguments to handle the projections into $W_{k}$ in (8.1), so we have that

$$
\left\|u-z_{k}\right\|+\left(\sum_{R \in \mathscr{E}_{k}}\left\|\nabla u-\nabla z_{k}\right\|_{R}^{2}\right)^{1 / 2} \leq C h_{k}\|f\|,
$$

and a duality argument can be used to show that

$$
\left\|u-z_{k}\right\| \leq C h_{k}^{2}\|f\|_{1} .
$$

This last result can also be derived easily from Theorems 6 and 7. Clearly, (6.9) implies that

$$
\left\|\zeta_{k}\right\| \leq C h_{k}^{2}\left(\|f\|+\left\|P_{W_{k}} z_{k}\right\|\right)
$$

and then (6.8) implies that

$$
\left\|P_{W_{k}} z_{k}\right\| \leq C^{\prime}\|f\| .
$$

Theorem 7 and an inverse inequality (see (A8) below) give (A3). We can derive (A2) similarly.

For our analysis, we introduce the conforming finite element space

$$
U_{k}=\left\{\xi \in C^{0}(\bar{\Omega}):\left.\xi\right|_{R} \in Q_{1,1}(R), \quad \forall R \in \mathscr{E}_{k} \text { and }\left.\xi\right|_{\partial \Omega}=0\right\} .
$$

Unlike the triangular case, $U_{k} \not \subset N_{k}$. Let $\tilde{z}_{k} \in U_{k}$ satisfy

$$
\tilde{a}_{k}\left(\tilde{z}_{k}, v\right)=\left(\tilde{f}_{k}, v\right), \quad \forall v \in U_{k} .
$$

The usual error estimate for this finite element method is

$$
\left\|u-\tilde{z}_{k}\right\|+h_{k}\left\|u-\tilde{z}_{k}\right\|_{k} \leq C h_{k}^{2}\|f\| .
$$

For each $k$, let $G_{k}$ be

$$
G_{k}=N_{k} \oplus\left\{v:\left.v\right|_{R}=a_{R}^{1} x y, a_{R}^{1} \in \mathbb{R}, \forall R \in \mathscr{E}_{k}\right\} ;
$$

$G_{k}$ contains both $N_{k}$ and $U_{k}$. Let $m_{k}=\operatorname{dim}\left(G_{k}\right)$. By the spectral theorem, there are eigenvalues $0<\lambda_{1} \leq \lambda_{2} \leq \cdots \leq \lambda_{m_{k}}$ and eigenfunctions $\phi_{1}, \phi_{2}, \cdots$, $\phi_{m_{k}} \in G_{k}$ such that

$$
\left(\phi_{i}, \phi_{j}\right)=\delta_{i, j} \quad \text { and } \quad \tilde{a}_{k}\left(\phi_{i}, v\right)=\lambda_{i}\left(\phi_{i}, v\right), \quad \forall v \in G_{k} .
$$

If $v \in G_{k}$, we write $v=\sum_{i=1}^{m_{k}} c_{i} \phi_{i}$ and define as in the standard case [2]

$$
\|\mid v\|_{s, k}=\left(\sum_{i=1}^{m_{k}} c_{i}^{2} \lambda_{i}^{s}\right)^{1 / 2} .
$$

The Cauchy-Schwarz inequality implies that

$$
\left|a_{k}(w, v)\right| \leq|||w|\left\|_{1+s, k}|| v\right\|_{1-s, k}
$$

for any $s \in \mathbb{R}$ and $v, u \in \in G_{k}$. Note that $\|\mid v\|_{0, k}=\|v\|$ and $\|v\|_{1, k}=\|v\|_{k}$. 
As mentioned in $\S 8$, we now modify the definition of $I_{k-1}^{k}$ so that it behaves well on $U_{k-1}$. So, let $I_{k-1}^{k}: G_{k-1} \rightarrow G_{k}$ be defined by (8.4) and

$$
\left.\sum_{i=1}^{4}(-1)^{i} I_{k-1}^{k} v\right|_{R}\left(p_{R, i}\right)=\left.\sum_{i=1}^{4}(-1)^{i} v\right|_{R}\left(p_{R, i}\right), \quad \forall R \in \mathscr{E}_{k},
$$

where $p_{R, i}$ are the vertices of $R$, labeled counterclockwise (i.e., so that $(-1)^{i}$ changes sign between the two ends of each edge of $\partial R$ ). As an immediate consequence of the definition, we have the following.

Proposition 1. If $\xi \in N_{k-1}$ and $v=x y$, then for any $R \in \mathscr{E}_{k}$ or $\mathscr{E}_{k-1}$,

$$
\begin{aligned}
& \left.\sum_{i=1}^{4}(-1)^{i} \xi\right|_{R}\left(p_{R, i}\right)=0, \\
& \left.\sum_{i=1}^{4}(-1)^{i} v\right|_{R}\left(p_{R, i}\right) \neq 0 .
\end{aligned}
$$

The first result guarantees that $I_{k-1}^{k}$ restricted to $N_{k-1}$ has the same definition as before. The second result guarantees that $I_{k-1}^{k}$ is well defined on $G_{k-1}$. We have the following technical lemma as in [3].

Lemma 4. There is a constant $C$ independent of $k$ such that

$$
\begin{aligned}
& C^{-1}\|v\|_{k} \leq\|v\|_{k-1} \leq C\|v\|_{k}, \quad \forall v \in C^{0}(\Omega), \\
& \|v\|_{k} \leq C h_{k}^{-1}\|v\|, \quad \forall v \in G_{k}, \\
& \left\|I_{k-1}^{k} v\right\| \leq C\|v\|, \quad \forall v \in G_{k-1} \\
& \tilde{I}_{k-1}^{k} v=v, \quad \forall v \in U_{k-1}, \\
& \left\|I_{k-1}^{k} \xi\right\|_{k} \leq C\|\xi\|_{k-1}, \quad \forall \xi \in N_{k-1} \oplus U_{k-1},
\end{aligned}
$$

where $I_{k-1}^{k}$ is defined by (8.4) and (A6).

Proof. Result (A7) is trivial. The $\mathscr{E}_{k}$ are quasi-uniform by construction, so (A8) is a standard inverse inequality. Result (A9) follows immediately from the definition of $I_{k-1}^{k}$. Since $U_{k-1} \subset U_{k} \subset G_{k}$ and $I_{k-1}^{k}$ is well defined, result (A10) follows trivially.

We easily obtain inequality (A11) for $\xi \in U_{k-1}$ from the definition of $I_{k-1}^{k}$ since $U_{k-1} \subset C^{0}(\Omega)$. Given $\xi \in N_{k-1} \oplus U_{k-1}$, define $v \in N_{k-1} \oplus U_{k-1}$, $w \in U_{k-1}$, and $z \in H_{0}^{1}(\Omega)$ by

$$
\begin{aligned}
& \tilde{a}_{k-1}(\xi, \zeta)=(v, \zeta), \quad \forall \zeta \in N_{k-1} \oplus U_{k-1}, \\
& \tilde{a}_{k-1}(w, \zeta)=(v, \zeta), \quad \forall \zeta \in U_{k-1}, \\
& \tilde{a}_{k-1}(z, \zeta)=(v, \zeta), \quad \forall \zeta \in H_{0}^{1}(\Omega) .
\end{aligned}
$$

Note that $\|z\|_{2} \leq C\|v\|$ by elliptic regularity, and that $\xi$ and $w$ are approximations to $z$ with the usual error estimates. It follows from the earlier results 
that

$$
\begin{aligned}
\left\|I_{k-1}^{k} \xi\right\|_{k} & \leq\left\|I_{k-1}^{k}(\xi-w)\right\|_{k}+C\|w\|_{k} \\
& \leq C\left[h_{k}^{-1}\left\|I_{k-1}^{k}(\xi-w)\right\|+\|\xi-w\|_{k-1}+\|\xi\|_{k-1}\right] \\
& \leq C\left[h_{k}^{-1}\|\xi-w\|+\|\xi\|_{k-1}\right] \\
& \leq C\left[h_{k}^{-1}(\|\xi-z\|+\|w-z\|)+\|\xi\|_{k-1}\right] \\
& \leq C\left[h_{k}\|v\|+\|\xi\|_{k-1}\right] .
\end{aligned}
$$

Finally, (A12) gives

$$
\|v\|^{2}=\tilde{a}_{k-1}(\xi, v) \leq\|\xi\|_{k-1}\|v\|_{k-1} \leq C h_{k}^{-1}\|\xi\|_{k-1}\|v\|,
$$

and (A11) follows.

We are in a position to prove that the $k$ th level iteration $\operatorname{MG}\left(k, g_{0}, \tilde{f}_{k}\right)$, when applied to the problem of finding $z \in N_{k}$ such that

$$
\tilde{a}_{k}(z, \xi)=\left(\tilde{f}_{k}, \xi\right), \quad \forall \xi \in N_{k},
$$

with the initial guess $g_{0}$ is a contraction in the energy norm. Let $e_{l}=z-g_{l} \in$ $N_{k}, l=0, \ldots, m$, where $g_{l}$ is defined as in (S), the smoothing step in (MG). Also let $e \in N_{k-1}$ and $\tilde{e} \in U_{k-1}$ satisfy

$$
\begin{aligned}
& \tilde{a}_{k-1}(e, \xi)=\tilde{a}_{k}\left(e_{m}, I_{k-1}^{k} \xi\right), \quad \forall \xi \in N_{k-1}, \\
& \tilde{a}_{k}(\tilde{e}, v)=\tilde{a}_{k}\left(e_{m}, I_{k-1}^{k} v\right), \quad \forall v \in U_{k-1} .
\end{aligned}
$$

Lemma 5. There is a constant $C$ such that

$$
\begin{aligned}
& \left\|e_{m}\right\|_{k} \leq C\left\|e_{0}\right\|_{k}, \\
& \left\|e_{m}\right\|_{2, k} \leq C h_{k}^{-1} m^{-1 / 2}\left\|e_{0}\right\|_{k}, \\
& \|e\|_{k-1} \leq C\left\|e_{0}\right\|_{k} .
\end{aligned}
$$

Proof. Equations (A15) and (A16) are proven using the ideas in [2]. It follows from the definition of the smoothing step $(S)$ that

$$
\left(e_{l}, \xi\right)=\left(e_{l-1}, \xi\right)-C_{1}^{-1} h_{k}^{2} \tilde{a}_{k}\left(e_{l-1}, \xi\right), \quad \forall \xi \in N_{k}
$$

If $e_{0}=\sum_{i=1}^{m_{k}} c_{i} \phi_{i}$, then

$$
e_{l}=\sum_{i=1}^{m_{k}} c_{i} \phi_{i}\left(1-C_{1}^{-1} h_{k}^{2} \lambda_{i}\right)^{l}, \quad l=0, \ldots, m,
$$

from which, and (A8) or (8.3), we have (A15). From this we can derive (A16) as in [2, equation (3.13)].

From (A13) and (A15), we see that

$$
\|e\|_{k-1}^{2}=\tilde{a}_{k}\left(e_{m}, I_{k-1}^{k} e\right) \leq \dot{\|} \mid e_{m}\left\|_{k}\right\| I_{k-1}^{k} e\left\|_{k} \leq C\right\| e_{m}\left\|_{k}\right\| e\left\|_{k-1} \leq C\right\| e_{0}\left\|_{k}\right\| e \|_{k-1},
$$

which yields inequality (A17).

Lemma 6. There is a constant $C$ such that

$$
\|e-\tilde{e}\|_{k-1} \leq C m^{-1 / 2}\left\|e_{0}\right\|_{k} .
$$


Proof. Let $f_{0} \in G_{k-1}$ satisfy

$$
\left(f_{0}, v\right)=\tilde{a}_{k}\left(e_{m}, I_{k-1}^{k} v\right), \quad \forall v \in G_{k-1} .
$$

We observe that

$$
\left\|f_{0}\right\|^{2}=\tilde{a}_{k}\left(e_{m}, I_{k-1}^{k} f_{0}\right) \leq\left\|| | e _ { m } | | | _ { 2 , k } \left|\left\|I_{k-1}^{k} f_{0}||_{0, k} \leq C\left|\left\|e_{m}||_{2, k}\right\| f_{0} \|,\right.\right.\right.\right.
$$

so that

$$
\left\|f_{0}\right\| \leq C\|\| e_{m} \|_{2, k} \text {. }
$$

Let $v_{0} \in H_{0}^{1}(\Omega) \cap H^{2}(\Omega)$ solve

$$
-\nabla \cdot\left(a \nabla v_{0}\right)+d v_{0}=f_{0} \quad \text { in } \Omega
$$

Note that, from the definition of $f_{0},(\mathrm{~A} 13)$, and (A14), $e$ and $\tilde{e}$ are approximations to $v_{0}$ in $N_{k-1}$ and $U_{k-1}$, respectively. Thus, as in (A3) and (A5), we see that

$$
\begin{aligned}
& \left\|v_{0}-e\right\|_{k-1} \leq C h_{k-1}\left\|f_{0}\right\|, \\
& \left\|v_{0}-\tilde{e}\right\|_{k} \leq C h_{k-1}\left\|f_{0}\right\|,
\end{aligned}
$$

and so, with (A16), we obtain

$$
\|e-\tilde{e}\|_{k-1} \leq C h_{k-1}\left\|f_{0}\right\| \leq C h_{k-1}\left\|e_{m}\right\|\left\|_{2, k} \leq C m^{-1 / 2}\right\| e_{0} \|_{k},
$$

completing the proof.

Lemma 7. There is a constant $C$ such that

$$
\left\|e_{m}-\tilde{e}\right\|_{k} \leq C m^{-1 / 2}\left\|e_{0}\right\|_{k} .
$$

Proof. From (A14) and (A10), we have

$$
\tilde{a}_{k}\left(e_{m}-\tilde{e}, v\right)=0, \quad \forall v \in U_{k-1} .
$$

By (A16), we get

$$
\begin{aligned}
\left\|e_{m}-\tilde{e}\right\|_{k}^{2} & =\tilde{a}_{k}\left(e_{m}-\tilde{e}, e_{m}-\tilde{e}\right) \\
& =\tilde{a}_{k}\left(e_{m}-\tilde{e}, e_{m}\right) \\
& \leq\|\| e_{m}-\tilde{e}\|\|_{0, k}\left\|e_{m}\right\|_{2, k} \\
& \leq C h_{k}^{-1} m^{-1 / 2}\left\|e_{m}-\tilde{e}\right\|\left\|e_{0}\right\|_{k} .
\end{aligned}
$$

Applying a duality argument to (A18), we can easily see that

$$
\left\|e_{m}-\tilde{e}\right\| \leq C h_{k}\left\|e_{m}-\tilde{e}\right\|_{k}
$$

and our result follows.

Lemma 8. There exist $\gamma \in(0,1)$ and an integer $m \geq 1$ in (MG), both independent of $k$, such that

$$
\left\|z-\operatorname{MG}\left(k, g_{0}, \tilde{f}_{k}\right)\right\|_{k} \leq \gamma\left\|z-g_{0}\right\|_{k} .
$$

Proof. We proceed by an induction argument on $k$. The result is trivial for $k=1$, even with $\gamma=0$. Let us suppose that the lemma is true for $k-1$. Lemmas 6 and 7 and (A11) imply that

$$
\begin{aligned}
\left\|z-\mathrm{MG}\left(k, g_{0}, \tilde{f}_{k}\right)\right\|_{k} & =\left\|e_{m}-I_{k-1}^{k} q_{p}\right\|_{k} \\
& \leq\left\|e_{m}-\tilde{e}\right\|_{k}+\left\|I_{k-1}^{k}(e-\tilde{e})\right\|_{k}+\left\|I_{k-1}^{k}\left(e-q_{p}\right)\right\|_{k} \\
& \leq C\left[m^{-1 / 2}\left\|e_{0}\right\|_{k}+\left\|e-q_{p}\right\|_{k-1}\right] .
\end{aligned}
$$



$N_{k-1}$,

By (A13) and the definition of the correction step (C) in (MG), for all $\xi \in$

$$
\begin{aligned}
\tilde{a}_{k-1}(e, \xi) & =\tilde{a}_{k}\left(z-g_{m}, I_{k-1}^{k} \xi\right) \\
& =\left(\tilde{f}_{k}, I_{k-1}^{k} \xi\right)-a_{k}\left(g_{m}, I_{k-1}^{k} \xi\right) \\
& =\left(\widehat{f}_{k}, \xi\right) ;
\end{aligned}
$$

therefore, $q_{j}=\mathrm{MG}\left(k-1, q_{j-1}, \widehat{f}_{k}\right)$, and the induction assumption and iteration gives

$$
\left\|e-q_{p}\right\|_{k-1} \leq \gamma^{p}\|e\|_{k-1}
$$

since $q_{0}=0$.

We obtain with (A17) that

$$
\left\|z-\mathrm{MG}\left(k, g_{0}, \tilde{f}_{k}\right)\right\|_{k} \leq C\left[m^{-1 / 2}\left\|e_{0}\right\|_{k}+\gamma^{p}\|e\|_{k-1}\right] \leq C_{2}\left(m^{-1 / 2}+\gamma^{p}\right)\left\|e_{0}\right\|_{k} \text {. }
$$

If $\gamma \in(0,1)$ is sufficiently small, then $C_{2} \gamma^{p}<\gamma / 2$ since $p>1$, and if $m$ is large enough, $C_{2} m^{-1 / 2}<\gamma / 2$. For such choices, we obtain the lemma.

Lemma 8 says that if the number of smoothing steps $m$ is large enough, the $k$ th-level iteration is a contraction. Let $R_{k}$ denote the standard interpolation operator for $U_{k}$. If $v \in H^{2}(\Omega)$, then

$$
\left\|v-R_{k} v\right\|+h_{k}\left\|v-R_{k} v\right\|_{k} \leq C h_{k}^{2}\|v\|_{2} .
$$

Proof of Lemma 2. From Lemma 8, (A3), (A19), and (A11), we see that

$$
\begin{aligned}
\left\|z_{k}-\widehat{z}_{k}\right\|_{k} & \leq \gamma^{r}\left\|z_{k}-I_{k-1}^{k} \widehat{z}_{k-1}\right\|_{k} \\
& \leq \gamma^{r}\left[\left\|z_{k}-u\right\|_{k}+\left\|u-R_{k-1} u\right\|_{k}+\left\|I_{k-1}^{k}\left(R_{k-1} u-\widehat{z}_{k-1}\right)\right\|_{k}\right] \\
& \leq C \gamma^{r}\left[h_{k}\|f\|+\left\|R_{k-1} u-\widehat{z}_{k-1}\right\|_{k-1}\right] \\
& \leq C \gamma^{r}\left[h_{k}\|f\|+\left\|R_{k-1} u-u\right\|_{k-1}+\left\|u-z_{k-1}\right\|_{k-1}\right. \\
& \leq C \gamma^{r}\left[h_{k}\|f\|+\left\|z_{k-1}-\widehat{z}_{k-1}\right\|_{k-1}\right] .
\end{aligned}
$$

Since $z_{1}-\widehat{z}_{1}=0$, iterating this expression leads to the inequality

$$
\left\|z_{k}-\widehat{z}_{k}\right\|_{k} \leq \sum_{j=1}^{k} C^{j} h_{k-j+1} \gamma^{j r}\|f\| \leq \frac{C_{3} \gamma^{r}}{1-2 C_{3} \gamma^{r}} h_{k}\|f\|,
$$

provided that $r$ is large enough so that $2 C_{3} \gamma^{r}<1$. Hence,

$$
\left\|z_{k}-\widehat{z}_{k}\right\|_{k} \leq C h_{k}\|f\|,
$$

which implies (8.7).

We prove (8.8) as in [17, Theorem 7.1, p. 162]. First, by (A3) and (A19),

$$
\begin{aligned}
\left\|z_{k}-I_{k-1}^{k} z_{k-1}\right\| & \leq\left\|z_{k}-u\right\|+\left\|u-R_{k} u\right\|+\left\|I_{k-1}^{k}\left(R_{k} u-z_{k-1}\right)\right\| \\
& \leq C h_{k}^{2}\|f\|_{1}+\left\|R_{k} u-z_{k-1}\right\| \\
& \leq C h_{k}^{2}\|f\|_{1} .
\end{aligned}
$$


Now Lemma 8 yields that

$$
\begin{aligned}
\left\|z_{k}-\widehat{z}_{k}\right\| & \leq \gamma^{r}\left\|z_{k}-I_{k-1}^{k} \widehat{z}_{k-1}\right\| \\
& \leq \gamma^{r}\left[\left\|z_{k}-I_{k-1}^{k} z_{k-1}\right\|+\left\|I_{k-1}^{k}\left(z_{k-1}-\widehat{z}_{k-1}\right)\right\|\right] \\
& \leq \gamma^{r}\left[C h_{k}^{2}\|f\|_{1}+\left\|z_{k-1}-\widehat{z}_{k-1}\right\|\right],
\end{aligned}
$$

so an induction argument yields (8.8). The proof is complete.

\section{BIBLIOGRAPHY}

1. D. N. Arnold and F. Brezzi, Mixed and nonconforming finite element methods: implementation, postprocessing and error estimates, RAIRO Modél. Math. Anal. Numér. 19 (1985), 7-32.

2. R. Bank and T. Dupont, An optimal order process for solving finite element equations, Math. Comp. 36 (1981), 35-51.

3. S. C. Brenner, An optimal-order multigrid method for $P 1$ nonconforming finite elements, Math. Comp. 52 (1989), 1-15.

4. __ A multigrid algorithm for the lowest-order Raviart-Thomas mixed triangular finite element method, SIAM J. Numer. Anal. 29 (1992), 647-678.

5. F. Brezzi, On the existence, uniqueness, and approximation of saddle point problems arising from Lagrangian multipliers, RAIRO Anal. Numér. 2 (1974), 129-151.

6. F. Brezzi, J. Douglas, Jr., R. Durán, and M. Fortin, Mixed finite elements for second order elliptic problems in three variables, Numer. Math. 51 (1987), 237-250.

7. F. Brezzi, J. Douglas, Jr., M. Fortin, and L. D. Marini, Efficient rectangular mixed finite elements in two and three space variables, RAIRO Modél. Math. Anal. Numér. 21 (1987), 581-604.

8. F. Brezzi, J. Douglas, Jr., and L. D. Marini, Two families of mixed finite elements for second order elliptic problems, Numer. Niath. 47 (1985), 217-235.

9. Zhangxin Chen, On the relationship between mixed and Galerkin finite element methods, $\mathrm{Ph}$. D. Thesis, Purdue University, West Lafayette, Indiana, 1991.

10. __ Unified analysis of the hybrid form of mixed finite elements for second order elliptic problems, J. Engrg. Math. 8 (1991), 91-102.

11. Analysis of mixed methods using conforming and nonconforming finite element methods, RAIRO Modél. Math. Anal. Numér. 27 (1993), 9-34.

12. Zhangxin Chen and B. Cockburn, Convergence of a finite element method for the drift diffusion semiconductor device equations: the multidimensional case, Numer. Math. (to appear).

13. Zhangxin Chen and J. Douglas, Jr., Prismatic mixed finite elements for second order elliptic problems, Calcolo 26 (1989), 135-148.

14. __ Approximation of coefficients in hybrid and mixed methods for nonlinear parabolic problems, Mat. Apl. Comput. 10 (1991), 137-160.

15. J. Douglas, Jr. and J. E. Roberts, Global estimates for mixed methods for second order elliptic problems, Math. Comp. 44 (1985), 39-52.

16. L. Gastaldi and R. Nochetto, Optimal $L^{\infty}$-error estimates for nonconforming and mixed finite element methods of lowest order, Numer. Math. 50 (1987), 587-611.

17. J. Mandel, S. McCormick, and R. Bank, Variational multigrid theory, Multigrid Methods (S. McCormick, ed.), SIAM, Philadelphia, PA, 1987, pp. 131-177.

18. L. D. Marini, An inexpensive method for the evaluation of the solution of the lowest order Raviart-Thomas mixed method, SIAM J. Numer. Anal. 22 (1985), 493-496.

19. J. C. Nedelec, Mixed finite elements in $\mathbf{R}^{3}$, Numer. Math. 35 (1980), 315-341.

20. A A new family of mixed finite elements in $\mathbf{R}^{3}$, Numer. Math. $\mathbf{5 0}$ (1986), 57-81. 
21. P. A. Raviart and J. M. Thomas, $A$ mixed finite element method for 2nd order elliptic problems, Mathematical Aspects of the Finite Element Method, Lecture Notes in Math., vol. 606, Springer-Verlag, Berlin, 1977, pp. 292-315.

22. R. Stenberg, $A$ family of mixed finite elements for the elasticity problem, Numer. Math. 53 (1988), 513-538.

Department of Computational and Applied Mathematics, Rice University, Houston, TEXAs 77251-1892

E-mail address: arbogast@rice.edu

University of Minnesota Army High Performance Computing Research Center, 1100 Washington Avenue South, Minneapolis, Minnesota 55415

Current address: Department of Mathematics and the Institute for Scientific Computation, Texas A\&M University, College Station, Texas 77843

E-mail address: zchen@isc.tamu.edu 